Research Paper

\title{
Information geometric superactivation of classical zero-error capacity of quantum channels
}

\author{
Laszlo GYONGYOSI ${ }^{1}$ and Sandor IMRE ${ }^{2}$ \\ ${ }^{1,2}$ Department of Telecommunications, Budapest University of Technology and Economics
}

\begin{abstract}
This paper introduces a fundamentally new method of finding the conditions for the superactivation of the zero-error capacity of quantum channels. The zero-error capacity of the quantum channel describes the amount of information which can be transmitted perfectly through a noisy quantum channel. The superactivation of the zero-error capacity of quantum channels makes it possible to use two quantum channels, each with zero zero-error capacity, with a positive joint zero-error capacity. Currently we have no theoretical background for describing all possible combinations of superactive quantum channels, hence there should be many other possible combinations. We give an algorithmic solution to the problem. To analyze the superactivation of the zero-error capacity, we introduce a new geometrical representation, called the quantum superball. Our method can be the first efficient algorithmic solution to discover the still unknown combinations to determine the superactivation of the zero-error capacity of quantum channels, without the extremely high computational costs.
\end{abstract}

\section{KEYWORDS}

Zero-error capacity, quantum channel, superactivation, zero-capacity quantum channel

\section{Introduction}

The zero-error capacity of the quantum channel measures the amount of information which can be transmitted through a noisy quantum channel with a zero probability of error. The superactivation of quantum channels may be the starting-point of a large-scale revolution in quantum information theory and in the communication of future quantum networks [1], [8]. The zeroerror capacity of the quantum channel can be a very important measure where perfect communication is required or the resources for communication are very limited. This capacity could have deep relevance in secure quantum communication, or in future quantum communication networks, where the quantum communication links can become permanently unavailable. The zero-error capacity cannot be calculated in an easy way such as in the case of classical, "non zero-error" capacity [9], [48], [49]. The computation of zero-error capac-

Received October 30, 2010; Revised December 12, 2010; Accepted December 28,2010

1) gyongyosi@hit.bme.hu, ${ }^{2)}$ imre@hit.bme.hu

DOI: 10.2201/NiiPi.2011.8.10 ity, for both the quantum and the classical case, is an extremely difficult computational problem [9].

This paper studies the superactivation property of the zero-error capacity of quantum channels, using very efficient informational geometric algorithmical tools. Since the revolutionary properties of superactivation of quantum channel capacities have been reported on, many new quantum informational results have been developed [1]-[3]. The superactivation of zero-error capacity implies the fact that a possible combination of quantum channels with zero zero-error capacity exists, where individually totally useless channels can activate each other, and their joint zero-error capacity will be greater than zero [2], [3].

The number of efficient approximation algorithms for quantum informational distances is very small because of the special properties of quantum informational generator functions and of asymmetric quantum informational distances. If we wish to analyze the properties of quantum channels using today's classical computer architectures, an extremely efficient algorithm is needed. Our method gives an algorithmic solution to 
the superactivation problem of the zero-error capacity of quantum channels. Recently, Duan and Cubitt et al. have found only one possible combination for superactivation of the classical zero-error capacity of quantum channels, and they have opened the debate on the existence of other possible channel combinations [2]-[6]. In this paper, we show that the currently known theoretical results can be verified in practice with our method, and our framework can be extended to discover other possible channels.

As has been shown by Medeiros et al. [7], [8] there are many unanswered questions remaining on the classical zero-error capacity of quantum channels. Moreover, the computation of the classical zero-error capacity of quantum channels is an extremely difficult and hard computational problem, as has been stated by Beigi et al. [9]. Currently we have no theoretical results for describing all possible combinations of superactive zero-capacity quantum channels, hence there should be many other possible combinations. As we will show, the result of Duan and Cubitt et al. [2], [3] is only one possible solution to superactivation of zero-error capacity and many other possible solutions can be discovered by our algorithmic solution. Our method provides a fundamentally new and efficient algorithmic solution for discovering all possible superactive quantum channels.

\subsection{Open questions on quantum capacities}

The problem of superactivation of zero-error capacity can be discussed as part of a larger problem set the problem of quantum channel additivity. The additivity problem is considered to be an important problem. By solving the additivity problem we can answer the question, is the zero-error capacity of a quantum communication channel affected by being restricted to non-entangled separable states in different quantum channel models? The quantum relative entropy function based on the interpretation of quantum channel capacity has been theoretically presented by HolevoSchumacher-Westmoreland [16], [17], later it has been studied by Cortese [10]. The capacity of a quantum channel has also been studied by Shor, who proved the equivalence of the additivity of quantum channel capacity and the additivity of minimum entropy output [39].

At present, conjectures connected to quantum channel additivity are still not solved, some have only been confirmed for some classes of quantum channel [33]. Different types of quantum channel have been studied by Brandao and Horodecki [34], King [35], Fukuda, King and Moser [36], Datta, Holevo and Suhov [37], Matsumoto and Yura [38], Wolf and Eisert [40], Ruskai, Szarek and Werner [12] and Schumacher and Westmoreland [16], [17]. Recently, the additivity property of a quantum channel has been studied by Smith [1], Hastings [33], and Brandao and Horodecki [34]. As shown in [33], the most basic questions on the classical capacity of a quantum channel still remain open. Open questions related to the superactivation of quantum channel capacities [1] can be discussed based on our novel and efficient informational geometric approach.

\section{The zero-error capacity of quantum channels}

Zero-error capacity has deep relevance in classical and quantum communication systems [47]-[49]. The zero-error capacity stands a very strong requirement in comparison to the standard capacity, since in the case of zero-error communication the error probability of the communication has to be zero, hence the transmission of information has to be perfect and no errors are allowed [8], [9].

As has been shown, there are many open questions and only one possible combination has been found to realize the superactivation of the zero-error capacity of quantum channels [3]. The zero-error capacity of the quantum channels can be a very important issue in some critical fields of future quantum communication networks, in which the errors cannot be tolerated. The zero-error capacity of the quantum channels is a very valuable field of quantum information processing, however the computation of this capacity is an extremely difficult problem in practice, both in classical system [48], [49], and both in a quantum system, as it has been shown by Beigi and Shor [9]. In the case of quantum channels the inputs can be product states and entangled states, which causes other difficulties in the computation of zero-error capacity. As also stated by Duan [2], the zero-error capacity of a quantum channel can be approached from the viewpoint of classical and quantum capacities.

The general view of the superactivation of quantum channels is illustrated in Fig. 1. Two quantum channels each with zero zero-error capacity, can be used to transmit classical information through a very noisy quantum communication channel. The superactivation of zeroerror capacity cannot be imagined for classical systems, and in the near future these results can revolutionize the communications over long-distance telecommunication, and it can help to enhance the security of quantum communication.

In Duan's work the superactive quantum channels could be superactivated after two uses [2]. Later, this result was improved by Cubitt et al. [3], since his combination required only a single use of the channel pair to realize the superactivation of zero-error capacities.

On the other hand, the entanglement among the input states is a required condition to realize the superactiva- 


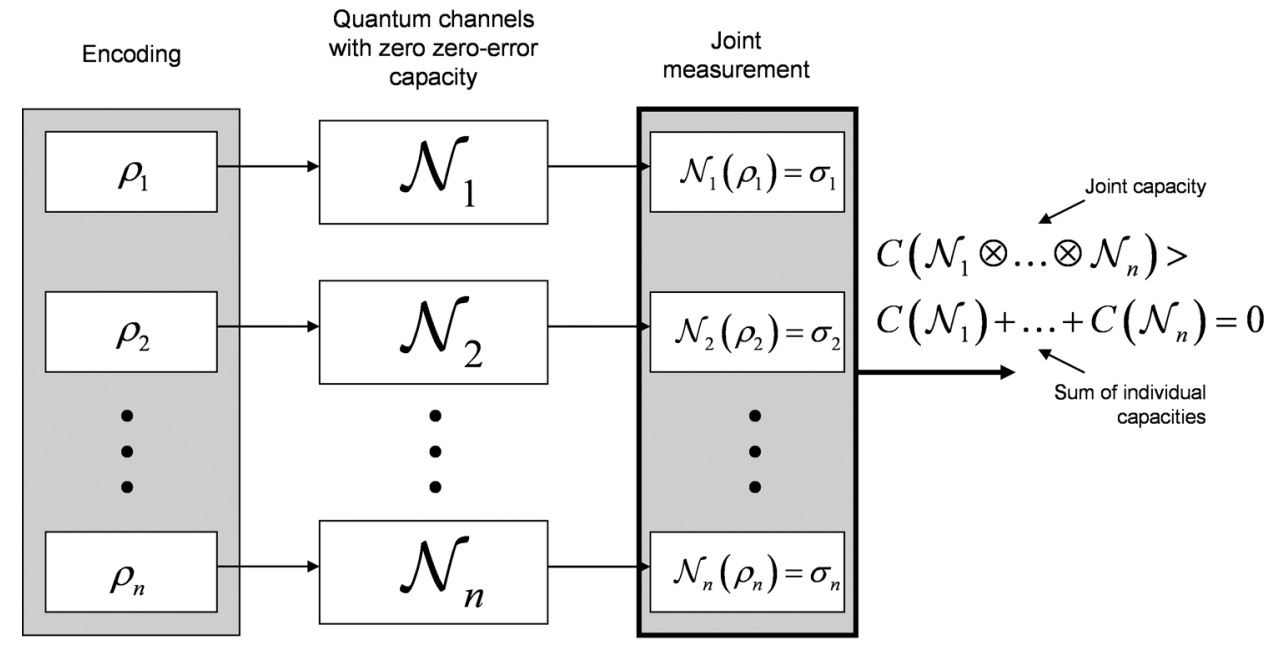

Fig. 1 Combination of quantum channels, with individual zero zero-error capacity, to realize perfect information transmission.

tion, otherwise the channels cannot be superactivated and the zero-error capacity will not be positive [3]. As also stated by Duan [2], superactivation can be achieved by using the entanglement, hence any classical messages can be transmitted perfectly through a zero errorcapacity channel, if the classical messages are encoded by entangled states. Duan and Cubitt et al. simultaneously studied the possibility of superactivation of the classical zero-error capacity of quantum channels [2][6], and concluded that a possible channel combination exists, in which one of the channels or each of the quantum channels can have zero classical zero-error capacity, however, the combinations of these kind of channels result in a positive classical zero-capacity. Hence the superactivation of the classical zero-error capacity of quantum channels is possible, although they only found one possible solution [2], [3].

The superactivation problem has been also studied by Smith [1], however in his work the superactivation of the quantum capacity of zero-capacity quantum channels has been analyzed instead of the zero-error capacity. In this paper we focus on the superactivation of asymptotic classical zero-error capacity of quantum channels which is currently a very important topic in quantum information theory, with still many open questions.

We give an extremely efficient algorithmical solution to discover the still undiscovered possible channel combinations for realize the superactivation of the classical zero-error capacity of quantum channels. Our work can be very valuable tools to a better understand of the properties of the classical zero-error capacity of quantum channels, and it can help to resolve the extremely difficult computational tasks regarding to the computing of zero-error capacity of quantum channels [9].

\subsection{Superactivation in the future's quantum communi- cations networks}

In many cases the error in the communication cannot be tolerated and the channel has to be totally errorfree. The zero-error information theory describes the maximum achievable rates and capacities, which are achieved in the case of a zero-error probability communication channel. The zero-error capacity was defined in Shannon's paper [47], later Bollobas [48] and Korner and Orlitsky [49] analyzed the mathematical and informational theoretic properties of zero-error capacity. In the case of future quantum communication networks, many new phenomena can be exploited, which were not available in the case of a classical communication networks. To use two zero-error capacity quantum links, we have to characterize only the input quantum states and the maps of the quantum channels, and after that has been done the two damaged or very noisy quantum links can be used to transmit information perfectly.

In future quantum communication networks, superactivation can be the ultimate weapon in situations where a quantum channel becomes totally or temporally unavailable due to damage to a link or a network communication problem, or where quantum communication channels are extremely noisy. With the help of the superactivation of zero-error capacity of quantum channels, the perfect information transmission can be realized in a very noisy network environment or in a damaged link. Moreover, it can be used to enhance the security of quantum communication over a very noisy 


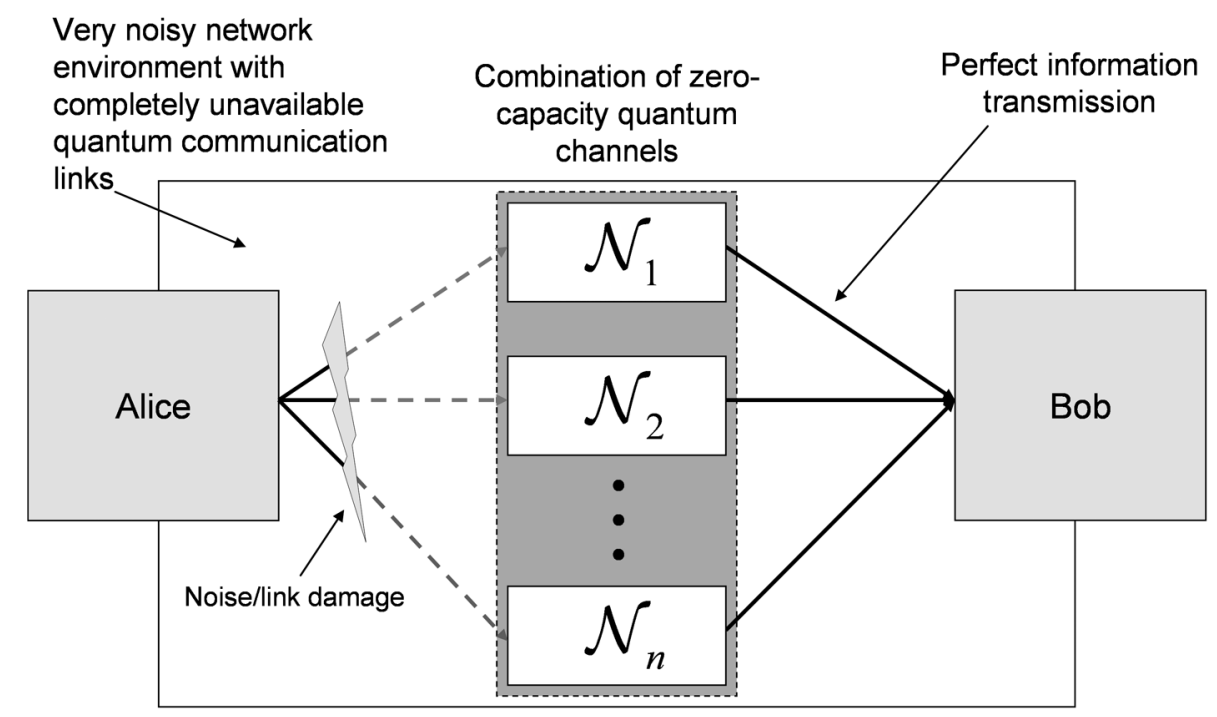

Fig. 2 The superactivation of zero-error capacity can help to transmit information through a very noisy quantum network, and the information can be transmitted perfectly through a temporarily unavailable quantum channel.

quantum environment, hence the application of superactivation in future quantum communication networks is very diverse.

In Fig. 2, we show possible ways of the applying superactivation in future quantum communication networks. Since the superactivation makes it possible to transmit information through a damaged quantum channel it can be used to avoid the temporarily unavailable status of network services.

Superactivation can be applied to an optical-fiber based optical quantum communication network [50], or in a free-space environment, both in a dense metropolitan area or over very long distances to improve the quality of information transmission. Our method can be a very valuable tool to realize noiseless quantum communication over a noisy communication environment. Using the superactivation of quantum channels, the effectiveness of the communication techniques in the future's quantum networks - in long-distances, or in a noisy metropolitan area - can be increased, and the currently used communication techniques can be revolutionized.

In addition to aiding metropolitan and long-distance quantum communication, the perfect information transmission through a quantum channel can have deep relevance both in military and secret government applications, or other cases where extremely high security is required.

\subsection{Theoretical results on the superactivation of zero- error capacity}

Cubitt et al. investigated an algebraic approach to study the superactivation property of the zero-error capacity, and as it has been found, there is also exists a stronger superactivation for the asymptotic zero-error capacity [3]. In their work, both quantum channels can have zero zero-error capacity, while in the case of Duan's method one of the channels has to be equipped with a greater than zero zero-error capacity, otherwise the superactivation would not have worked. Duan has found only one class of quantum channels with zero zero-error capacity for which the entangled input states can superactivate their capacities. The usage of entanglement also implies the fact that superactivation is not possible in the case of classical communication channels. Duan has also stated, that besides the fact that the classical zero-error capacity of quantum channels is superactive, the quantum zero-error capacity of a quantum channel is also superactive, hence there is an overlapping with the results of Smith [1]. We note, he has studied the quantum capacity of quantum channels instead of the quantum zero-error capacity.

Our method can be used to verify both the results of Duan and, the stronger conditions of Cubitt et al. In future work we would like to extend our method to analyze the superactivation of the asymptotic quantum capacity of zero-capacity quantum channels, which is still an open problem in quantum information theory [1]. 


\subsection{Geometrical analysis of zero-error capacity}

This paper introduces a completely revolutional algorithmical approach to find the still undiscovered combinations for realizing superactivation of the classical zero-error capacity of quantum channels. The proposed geometrical method is based on the HolevoSchumacher-Westmoreland (HSW) channel capacity and its geometrical interpretation [10], [16]-[18]. In our work, we apply computational geometry in quantum space, between pure and mixed quantum states. Using extremely fast computational geometric methods the superactivation of asymptotic zero-error capacity quantum channels can be efficiently analyzed with the help of currently available classical computational architectures.

Computational Geometry was originally focused on the construction of efficient algorithms and provides a very valuable and efficient tool for computing hard tasks. In many cases, traditional linear programming methods are not very efficient. In this paper, advanced geometric methods play a fundamental role in the analysis of the superactivation of zero-error capacity quantum channels. To analyze a quantum channel for a large number of input quantum states with classical computer architectures, very fast and efficient algorithms are required [44]-[46].

In Fig. 3, we illustrate the logical structure of the analysis and the cooperation of classical and quantum systems. We would like to analyze the properties of quantum channels using current classical computer architectures - since, currently we have no quantum computers - and the most efficient currently available algorithms. To this day, the most efficient classical algorithms for this purpose are computational geometric methods. We use these classical computational geometric tools to discover the still unknown "superactive" zero-error capacity quantum channels.

In this paper, we will apply the methods of computational geometry to analyze the superactivation of zero-error capacity quantum channels, however we will use quantum information as a distance measure instead of classical geometric distances. Unlike ordinary geometric distances, the quantum informational distance is not a metric and is not symmetric, hence this pseudodistance features as a measure of informational distance. We combine the models of information geometry and the fast methods of computational geometry, as depicted in Fig. 4.

At present, computational geometry algorithms are an active, widely used and integrated research field. Many difficult problems can be extended to computational geometric methods, however these geometric problems require well-designed and efficient algorithms [10], [14], [18]. Recently, the possibilities of

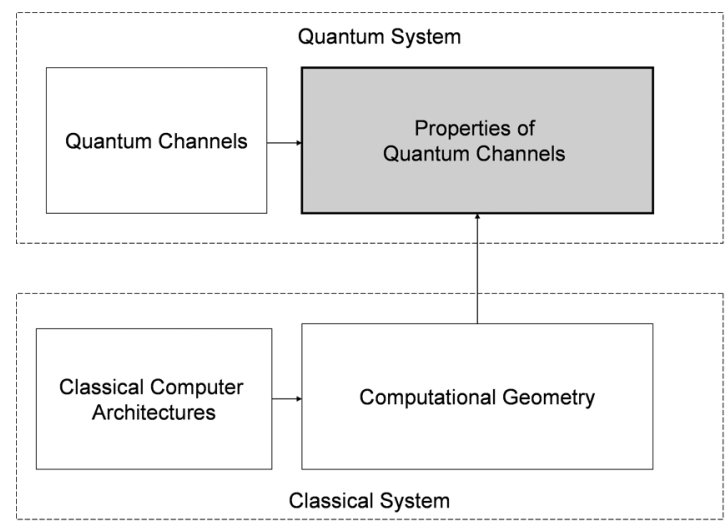

Fig. 3 The logical structure of our analysis. We use current classical architectures to analyze the properties of quantum channels.

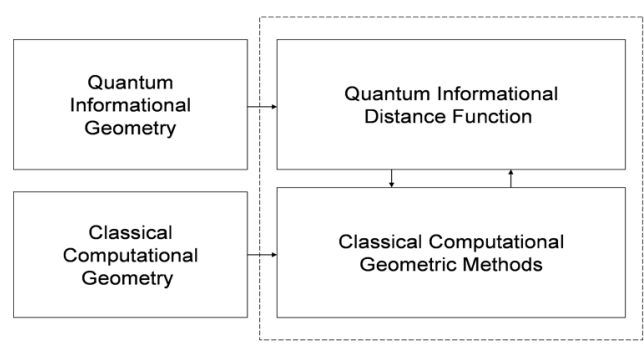

Fig. 4 Quantum information as distance measure in classical computational geometric methods.

the application of computational geometric methods in quantum space have been studied by Kato et al. [26] and Nielsen et al. [30]. Nielsen has shown very useful and efficient geometrical approaches to compute the Voronoi diagrams between the quantum states [28], [29], [31], [32].

\subsection{Problem of superactivation}

To this day, strict additivity of quantum channel capacity has been conjectured, but not proven. The property is known to hold for some special cases, but the generalized rule is still unknown. As depicted in Fig. 5, the problem of superactivation of zero-error capacity quantum channels can be viewed as a smaller subset of a larger problem set involving the additivity of quantum channels and the superactivation of zero-capacity quantum channels.

We show that a modified version of Chen's weak coreset method [20] and Ackermann's modified clustering algorithm [19] can be applied to quantum informational distances. Then the superactivation property of different quantum channel models with various channel 
probabilities can be analyzed extremely fast in our fundamentally new framework. Our iterations are based on the computed radius of a superball, the iterations are made on the channel input states, channel models, and channel parameters. The output of the algorithm is the radius of the quantum informational superball.

In our model, the results of Duan and Cubitt et al. on superactivation of zero-capacity channels is only one possible solution to the problem of searching for "superactive" quantum channels. Our method can be extended to every possible quantum channel model and channel parameter. Using the coreset method [20], we can analyze the capacities of quantum channels, for an extremely large input set and for all possible channel models with extremely high efficiency. We show that the modified version of Chen's algorithm can be applied in quantum space to quantum informational distances and the performance of the iteration can be dramatically accelerated by the introduction of weak coresets and $\mu$-similar quantum informational distances. We use the modified version of Ackermann's clustering algorithm [19] for weak coresets, hence the $k$-median problem and the radius of the super quantum informational ball can be computed very fast with respect to quantum informational distances.

The presented algorithm has less complexity than other existing coreset and approximation algorithms, which can also be applied in quantum space. In classical systems, the Kullback-Leibler distance is used as a distance metric in many fields, such as statistics, mathematics, speech recognition and informational databases. The Kullback-Leibler distance is a more useful distance metric in these cases than the ordinary Euclidean distance, since we have to compare

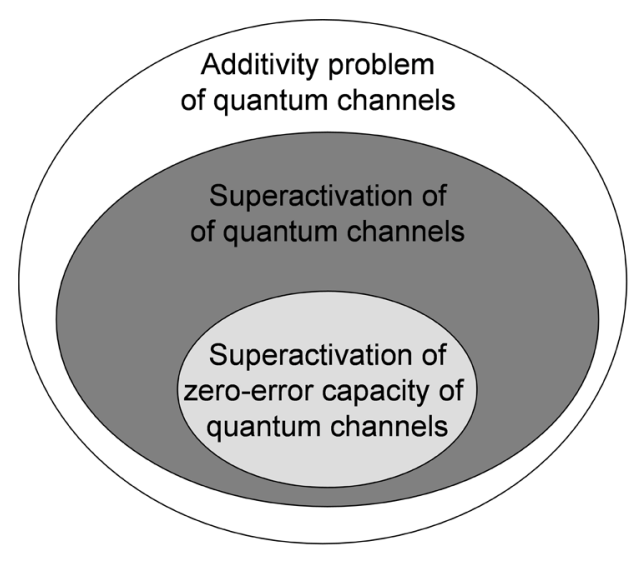

Fig. 5 The problem of superactivation of zero-error capacity of quantum channels as a sub domain of larger problem sets. probability distributions, speech samples, images, time series or other matrices, etc. [20].

\subsection{Median quantum states in quantum space}

We will use the well-known coreset method in quantum space between quantum states and we will show that these methods can be implemented very efficiently to analyze the properties of quantum channels. The coreset approach can be extended to the $k$-median clustering of channel output states. The construction of coresets has been studied in classical systems in [15], [22], [23]. In classical computational geometry, coreset algorithms are well-known and widely applied methods. The coreset method generates a small weighted set from the original dataset, such that the smaller set has the same properties as the original, larger set. The coreset method can be applied to analyze the properties of quantum channels for a large set of output channel states, since coreset methods have the same cost for clustering as the original larger set, with an approximation error $\varepsilon$. Hence, the structural properties of quantum channels can be approximated well by a much smaller set, called the coreset. Coreset methods were studied by Har-Peled and Kushal [23] who have found that the size of the coreset is independent of the size of the input set, however it still has an exponential dependence on the dimension parameter $d$. Later, Chen has shown [20] that the size of the coreset can be a linear function of $d$, so it can be applied to higher dimensions with great efficiency. After Chen's work, Feldman [25] introduced the definition of weak coresets, which differs a little from the coreset method as defined originally. The properties of Voronoi diagrams [13] in quantum space have been studied by Kato et al. [26] and Nielsen et al. [24], [29], [30] however the problem of clustering was not analyzed in their work. The coreset method for different distances has been studied in the literature. Euclidean methods were studied in [21], [22] and a non-Euclidean metric by Banerjee [27], Nielsen [24] and Ackermann [19].

In our paper, we will define a coreset approach between quantum states in quantum space to analyze the channel output states in terms of a smaller set generated by the coreset method. The input and the output of the coreset algorithm are illustrated in Fig. 6.

The output of the joint measurement of two channels is the input of the proposed geometrical approach. The geometrical method computes the joint capacity, based on the clustering of channel output quantum states and a convex hull calculation. The output of the constructed method is the radius of the smallest quantum informational superball. 


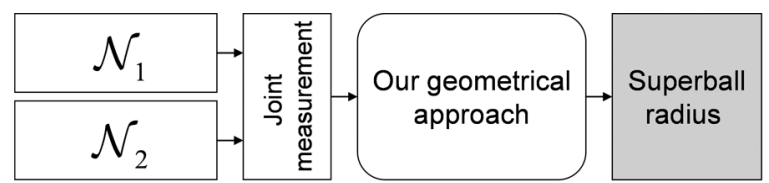

Fig. 6 The superactivation of the zero-error capacity is analyzed in terms of the radius of the quantum superball, which is computed by our geometrical approach.

\section{Communication over quantum chan- nels}

Before we start to discuss the properties of zero-error capacity of quantum channels, in this section we give a brief overview on the general properties of communication over such channels.

\subsection{The general classical communication mode}

In the classical communication model, the sender and receiver can be modeled by random variables $X=$ $\left\{p_{i}=P\left(x_{i}\right)\right\}, i=1, \ldots N$ and $Y=\left\{p_{i}=P\left(y_{i}\right)\right\}$, $i=1, \ldots N$. In classical systems, the Shannon entropy of the discrete random variable $X$ is denoted by $H(X)$ and can be defined as $H(X)=-\sum_{i=1}^{N} p_{i} \log \left(p_{i}\right)$.

For conditional random variables, the probability of random variable $X$ given $Y$ is denoted by $p(X \mid Y)$. The noise in the channel increases the uncertainty in $X$, given Bob's output $Y$. The informational theoretic noise of the channel increases the conditional Shannon entropy $H(X \mid Y)$, defined as $H(X \mid Y)=\sum_{i=1}^{N_{X}} \sum_{j=1}^{N_{Y}} p\left(x_{i}, y_{j}\right) \log p\left(x_{i} \mid y_{j}\right)$, thus the radius of the smallest enclosing quantum informational ball will decrease for fixed $H(X)$ [11].

The general classical informational theoretic model for a noisy quantum channel is illustrated in Fig. 7. Our geometrical analysis is focused on the mixed quantum state, received by Bob. Alice's pure state is denoted by $\rho_{A}$, the noise is modeled by an affine map $\mathcal{N}$ and Bob's mixed input state is denoted by $\mathcal{N}\left(\rho_{A}\right)=\sigma_{B}$. For random variables $X$ and $Y, H(X, Y)=H(X)+H(Y \mid X)$, where $H(X), H(X, Y)$ and $H(Y \mid X)$ are defined by probability distributions. We measure in a geometrical representation the information which can be transmitted in the presence of noise on the quantum channel.

In the classical communication model, we seek to maximize $H(X)$ and minimize $H(X \mid Y)$ in order to maximize the radius of the smallest enclosing ball of Bob, since the radius can be computed as

$$
r^{*}=\max _{\left\{\text {all possible } x_{i}\right\}} H(X)-H(X \mid Y) \text {. }
$$

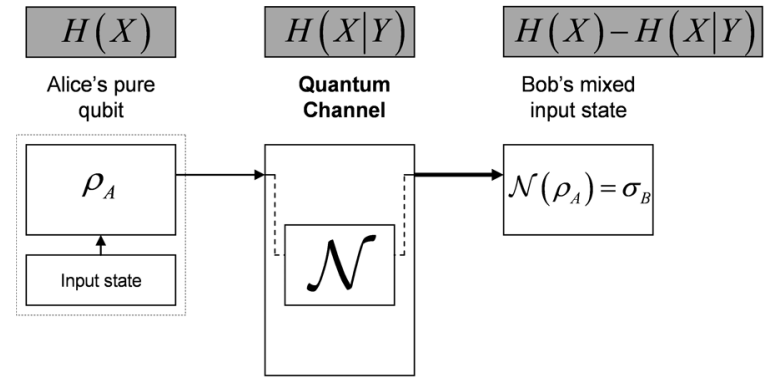

Fig. 7 The classical communication model.

To compute the radius $r^{*}$ of the smallest informational ball of quantum states and the entropies between mixed quantum states, instead of the classical Shannon entropy, we use the Holevo-Schumacher-Westmoreland (HSW) channel capacity [16], [17].

Geometrically, the presence of noise on the quantum channel causes a detectable mapping to change from a noiseless one-to-one relationship to a stochastic map. In the classical model of a quantum channel, the input is in a pure state denoted by $\left\{p_{i}, \rho_{i}\right\}$ and a measurement is made at the end of the quantum channel, which extracts the classical information from the sent quantum state. The image of the quantum channel's linear transform $\mathcal{N}$ is an ellipsoid. To preserve the condition for a density matrix $\rho$, the noise on the quantum channel $\mathcal{N}$ must be trace-preserving, i.e. $\operatorname{Tr} \mathcal{N}(\rho)=\operatorname{Tr}(\rho)$ and it must be completely positive, i.e. for any identity map $I$, the map $\mathcal{N} \otimes I$ maps a semi-positive Hermitian matrix to a semi-positive Hermitian matrix. A quantum channel $\mathcal{N}$ can be described by an affine map, which maps quantum states to other quantum states. Geometrically, the effect of the transformation $\mathcal{N}$ maps the Bloch ball to a deformed ball contained inside the Bloch ball.

\subsection{Holevo-Schumacher-Westmoreland channel ca- pacity}

In our work, we use the Holevo-SchumacherWestmoreland (HSW) channel capacity [16], [17] to analyze the superactivation property of quantum channels. According to the HSW theorem, the single use capacity $C^{(1)}(\mathcal{N})$ of a quantum channel $\mathcal{N}$, can be defined as follows [16], [17]:

$$
\begin{aligned}
C^{(1)}(\mathcal{N})= & \max _{\left\{\text {all possible } p_{i} \text { and } \rho_{i}\right\}} \mathcal{X}_{\text {output }} \\
= & \max _{p_{1}, \ldots, p_{n}, \rho_{1}, \ldots, \rho_{n}} \mathrm{~S}\left(\mathcal{N}\left(\sum_{i=1}^{n} p_{i}\left(\rho_{i}\right)\right)\right) \\
& +\sum_{i=1}^{n} p_{i} \mathrm{~S}\left(\mathcal{N}\left(\rho_{i}\right)\right),
\end{aligned}
$$


where $\mathcal{X}_{\text {output }}$ is the Holevo quantity of the output, $\mathrm{S}(\rho)=-\operatorname{Tr}(\rho \log \rho)$ is the von Neumann entropy, and $\mathcal{N}\left(\rho_{i}\right)$ represents the output density matrix obtained from the quantum channel input density matrix $\rho_{i}$ [16]. Using the result of the HSW theorem [16], we will refer to the single use channel capacity as the radius of the smallest enclosing ball as follows:

$$
r^{*}=C^{(1)}(\mathcal{N})=\max _{\left\{\text {all possible } p_{i} \text { and } \rho_{i}\right\}} \mathcal{X}_{\text {output }} .
$$

In this paper, we use the geometrical interpretation of HSW channel capacity, using quantum relative entropy as a distance measure function. The HSW channel capacity can be defined by using the quantum relative entropy function as a distance measure. We will show that the superactivation of zero-error capacity quantum channels can be analyzed in a geometrical representation, which uses the quantum relative entropy-based HSW channel capacity.

\subsubsection{Geometrical interpretation of HSW channel ca- pacity}

We use the results of Schumacher and Westmoreland to describe geometrically the channel capacity of quantum channels in terms of quantum relative entropy. The authors of [16] have shown that the capacity of a quantum channel can be measured geometrically, using quantum relative entropy function as a distance measure. Schumacher and Westmoreland have shown that the channel capacity of every optimal output state $\rho_{k}$ can be expressed as [16]

$$
C^{(1)}(\mathcal{N})=D\left(\rho_{k} \| \sigma\right),
$$

where $\sigma=\sum p_{k} \rho_{k}$ is the optimal average output state and the relative entropy function of two density matrices can be defined as

$$
D\left(\rho_{k} \| \sigma\right)=\operatorname{Tr}\left[\rho_{k} \log \left(\rho_{k}\right)-\rho_{k} \log (\sigma)\right] .
$$

In this definition, $T r$ is the trace operator. In conclusion, for non-optimal output states $\delta$ and optimal average output state $\sigma=\sum p_{k} \rho_{k}$, we have $C^{(1)}(\mathcal{N})=$ $D(\delta \| \sigma) \leq D\left(\rho_{k} \| \sigma\right)$. Moreover, in [16], Schumacher and Westmoreland have also shown that there exists at least one optimal output state $\left\{p_{k}, \rho_{k}\right\}$ which achieves the optimal capacity $C^{(1)}(\mathcal{N})=D\left(\rho_{k} \| \sigma\right)$. The geometrical interpretation of quantum channel capacity was introduced in [16], using the quantum relative entropy function as a distance measure as follows:

$$
C^{(1)}(\mathcal{N})=r^{*}=\min _{\{\sigma\}} \max _{\{\rho\}} D(\mathcal{N}(\rho) \| \mathcal{N}(\sigma)) .
$$

If we define the convex hull of possible channel output states for channel $\mathcal{N}$ as $\mathcal{A}$ and the convex hull of the set of states as $\mathcal{B}$, then for $\mathcal{A} \in \rho$ and $\mathcal{B} \in \sigma$ :

$$
C^{(1)}(\mathcal{N})=r^{*}=\min _{\{\sigma\}} \max _{\{\rho\}} D(\rho \| \sigma) .
$$

Schumacher and Westmoreland have also proven in [16], that there exists an optimum output state $\left\{p_{k}, \rho_{k}\right\}$ for every $\sigma$ that satisfies the maximization, such that $\sigma=\sum p_{k} \rho_{k}$. They have also shown that the average output state $\sigma=\sum p_{k} \rho_{k}$ which maximizes the capacity for any optimal set of output states $\rho=\sum\left\{p_{k}, \rho_{k}\right\}$ is unique [10], [16]. We analyze the superactivation of the quantum channel by clustering and convex hull calculations based on quantum relative entropy. If we denote the optimal output states by $\left\{\mathcal{N}\left(\psi_{k}\right)=p_{k}, \rho_{k}\right\}$ which achieve the capacity $C^{(1)}(\mathcal{N})$ of channel $\mathcal{N}$ and $\sigma=\sum_{k} p_{k} \rho_{k}$, then the single use quantum channel capacity can be derived in terms of the quantum relative entropy in the following way [16], [17]:

$$
\begin{aligned}
& \sum_{k} p_{k} D\left(\rho_{k} \| \sigma\right) \\
= & \sum_{k}\left(p_{k} \operatorname{Tr}\left[\rho_{k} \log \left(\rho_{k}\right)\right]-p_{k} \operatorname{Tr}\left[\rho_{k} \log (\sigma)\right]\right) \\
= & \sum_{k}\left(p_{k} \operatorname{Tr}\left[\rho_{k} \log \left(\rho_{k}\right)\right]\right)-\operatorname{Tr}\left[\sum_{k}\left(p_{k} \rho_{k} \log (\sigma)\right)\right] \\
= & \sum_{k}\left(p_{k} \operatorname{Tr}\left[\rho_{k} \log \left(\rho_{k}\right)\right]\right)-\operatorname{Tr}[\sigma \log (\sigma)] \\
= & \mathrm{S}(\sigma)-\sum_{k} p_{k} \mathrm{~S}\left(\rho_{k}\right)=\mathcal{X} .
\end{aligned}
$$

It can therefore be concluded that the Holevo quantity $\mathcal{X}$ can be expressed in terms of the quantum relative entropy and the $C^{(1)}(\mathcal{N})$ single use HSW channel capacity as [16]

$$
C^{(1)}(\mathcal{N})=\max _{\left\{\text {all } p_{k}, \psi_{k}\right\}} \sum_{k} p_{k} D\left(\mathcal{N}\left(\psi_{k}\right) \| \mathcal{N}(\psi)\right),
$$

where $\psi_{k}$ denotes the input quantum states of channel $\mathcal{N}$ and $\psi=\sum_{k} p_{k} \psi_{k}$. The geometric interpretation of the HSW channel capacity has been studied by Cortese [10], who also extended these results to the general qudit channels.

\subsection{Asymptotic HSW channel capacity and quantum superball representation}

Using the resulting quantum relative entropy function [16] and the HSW-theorem, the $C(\mathcal{N})$ asymptotic classical capacity of the quantum channel can be expressed with the help of the radii of the smallest quantum informational balls as follows:

$$
r_{\text {super }}^{*}(\mathcal{N})=C(\mathcal{N})=\lim _{n \rightarrow \infty} \frac{1}{n} C^{(1)}\left(\mathcal{N}^{\otimes n}\right)
$$




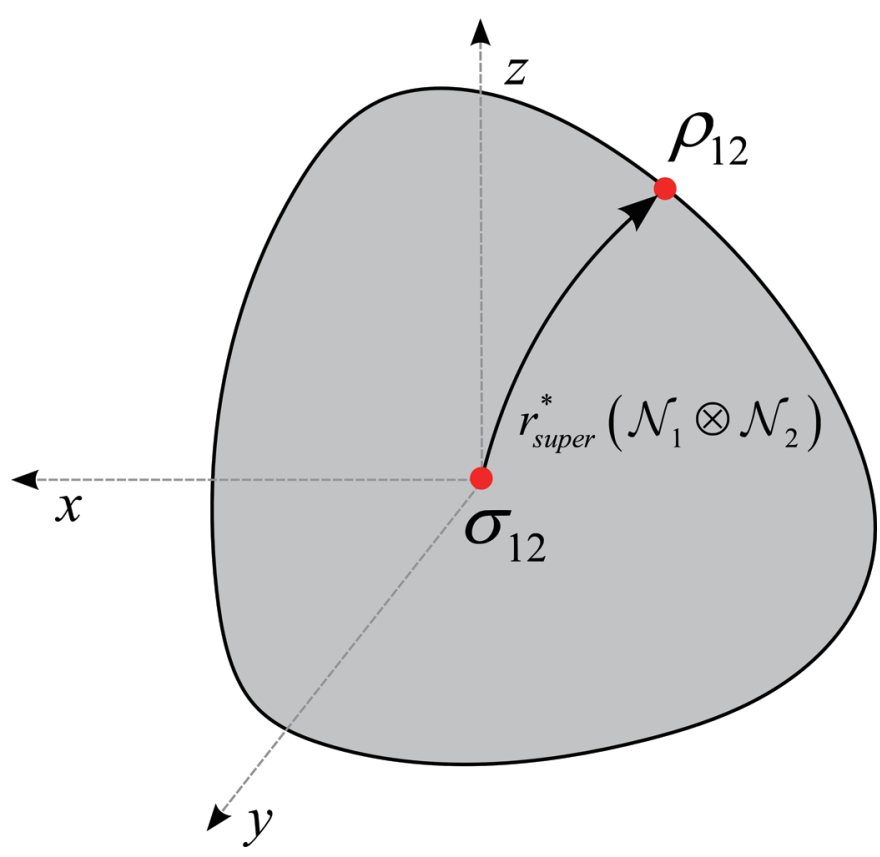

Fig. 8 The superactivation of the zero-error capacity is analyzed by the quantum informational superball.

$$
\begin{array}{r}
=\lim _{n \rightarrow \infty} \frac{1}{n}\left(\sum_{i=1}^{n} r_{i}^{*}\right) \\
=\lim _{n \rightarrow \infty} \frac{1}{n} \max _{p_{1}, \ldots, p_{n}, \rho_{1}, \ldots, \rho_{n}}\left(\mathcal{X}_{A B}\right)^{\otimes n} \\
=\lim _{n \rightarrow \infty} \frac{1}{n} \sum_{n}\left(\min _{\sigma_{1 \ldots n}} \max _{\rho_{1 \ldots n}}\right. \\
\left.\quad \times D\left(\rho_{k}^{A B} \| \sigma^{A B}\right)\right),
\end{array}
$$

where $r_{i}^{*}$ is the single use capacity of the $i$-th use of quantum channel $\mathcal{N}, \rho_{k}^{A B}$ is the optimal output channel state, and $\sigma^{A B}$ is the average state. We analyze the superactivation property of the quantum channel, using the mini-max criterion for states $\rho_{k}^{A B}$ and $\sigma^{A B}$. The radius $r_{\text {super }}^{*}$ of the superball is equal to the asymptotic classical capacity.

In the superactivation problem, we have to use different quantum channel models [1]. For two different quantum channels $\mathcal{N}_{1}^{\otimes n}$ and $\mathcal{N}_{2}^{\otimes n}$, the asymptotic HSW channel capacity $C\left(\mathcal{N}_{1} \otimes \mathcal{N}_{2}\right)$ is equal to the sum of the radii $r_{\text {super }}^{*}\left(\mathcal{N}_{1}\right)$ and $r_{\text {super }}^{*}\left(\mathcal{N}_{2}\right)$ of the quantum informational superballs, whose radii form a new quantum superball with radius

$$
\begin{aligned}
& r_{\text {super }}^{*}\left(\mathcal{N}_{1} \otimes \mathcal{N}_{2}\right)=C\left(\mathcal{N}_{1} \otimes \mathcal{N}_{2}\right) \\
& =\lim _{n \rightarrow \infty} \frac{1}{n} C^{(1)}\left(\left(\mathcal{N}_{1} \otimes \mathcal{N}_{2}\right)^{\otimes n}\right) \\
& =r_{\text {super }}^{*}\left(\mathcal{N}_{1}\right)+r_{\text {super }}^{*}\left(\mathcal{N}_{2}\right) .
\end{aligned}
$$

In Fig. 8, we illustrate the superball representation for the analysis of two quantum channels, however it naturally can be extended to $n$ different quantum channel models.

The quantum informational ball has a distorted geometrical structure, and the elements of classical Euclidean geometry, such as the classical Euclidean distance functions, cannot be used. It makes the computation of the quantum ball a much harder computational task, but on the other hand, efficient geometrical functions can be constructed for this purpose.

In Fig. 9, we summarize our geometrical iteration process. The inputs of the rounded box are the channel output states of two quantum channels $\mathcal{N}_{1}^{\otimes n}$ and $\mathcal{N}_{2}^{\otimes n}$. The joint channel construction is denoted by $\left(\mathcal{N}_{1}+\mathcal{N}_{2}\right)^{\otimes n}$, the output of the rounded box is the radius $r_{\text {super }}^{*}\left(\mathcal{N}_{1} \otimes \mathcal{N}_{2}\right)$ of the quantum informational superball.

The output of the algorithm is the quantum informational superball, which ball is computed by efficient informational geometrical approaches. The quantum superball can be obtained after the joint measurement of the quantum channels. The superactivation property is analyzed by the length of the radius of the quantum superball.

The recursive iterations are made on the following parameters: the maps of the quantum channels, the probabilities of the channel combinations and the type of the input states. According to the length of the superball radius, the iteration stops if the conditions for 


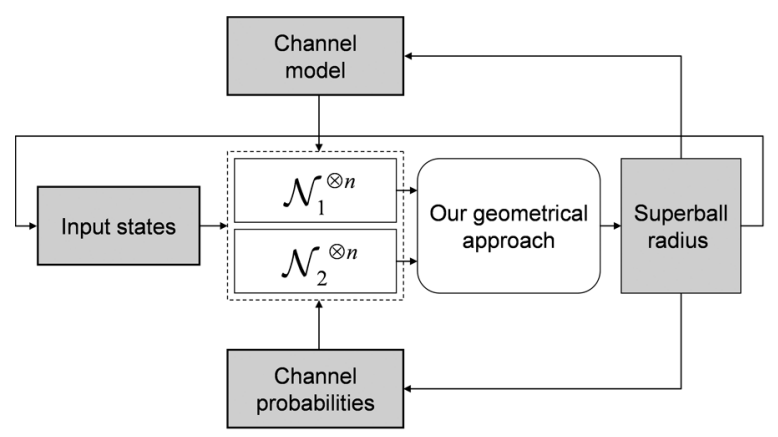

Fig. 9 The recursive algorithm used to find a combination for which superactivation holds.

superactivation hold.

The problem of clustering in quantum space, using the quantum informational distance as a distance function, is a completely new area in quantum information theory.

\section{Geometrical interpretation of quan- tum informational distance}

The quantum entropy $\mathbf{S}(\rho)$ is equal to the Shannon entropy for the eigenvalue distribution $\mathrm{S}(\rho)=\mathrm{S}(\lambda)=$ $-\sum_{i=1}^{d} \lambda_{i} \log \lambda_{i}$, where $d$ is the level of the quantum system. The relative entropy in classical systems is a measure that quantifies how close a probability distribution $p$ is to a model or candidate probability distribution $q$ [24]. For $p$ and $q$ probability distributions, the relative entropy is given by $D(p \| q)=\sum_{i} p_{i} \log _{2} \frac{p_{i}}{q_{i}}$, while the relative entropy between quantum states is measured by

$$
D(\rho \| \sigma)=\operatorname{Tr}[\rho(\log \rho-\log \sigma)] .
$$

The quantum informational distance has some distance-like properties, however it is not commutative [24], thus $D(\rho \| \sigma) \neq D(\sigma \| \rho)$, and $D(\rho \| \sigma) \geq 0$ iff $\rho \neq \sigma$, and $D(\rho \| \sigma)=0$ iff $\rho=\sigma$. As it has been stated by Cortese [10], the quantum relative entropy for general quantum state $\rho=(x, y, z)$ and mixed state $\sigma=(\tilde{x}, \tilde{y}, \tilde{z})$, with radii $r_{\rho}=\sqrt{x^{2}+y^{2}+z^{2}}$ and $r_{\sigma}=\sqrt{x^{2}+y^{2}+z^{2}}$ is given by

$$
\begin{aligned}
D(\rho \| \sigma)= & \frac{1}{2} \log \frac{1}{4}\left(1-r_{\rho}^{2}\right) \\
& +\frac{1}{2} r_{\rho} \log \frac{\left(1+r_{\rho}\right)}{\left(1-r_{\rho}\right)}-\frac{1}{2} \log \frac{1}{4}\left(1-r_{\sigma}^{2}\right) \\
& -\frac{1}{2 r_{\sigma}} \log \frac{\left(1+r_{\sigma}\right)}{\left(1-r_{\sigma}\right)}\langle\rho, \sigma\rangle,
\end{aligned}
$$

where $\langle\rho, \sigma\rangle=(x \tilde{x}+y \tilde{y}+z \tilde{z})$. For a maximally mixed state $\sigma=(\tilde{x}, \tilde{y}, \tilde{z})=(0,0,0)$ and $r_{\sigma}=0$, the quantum

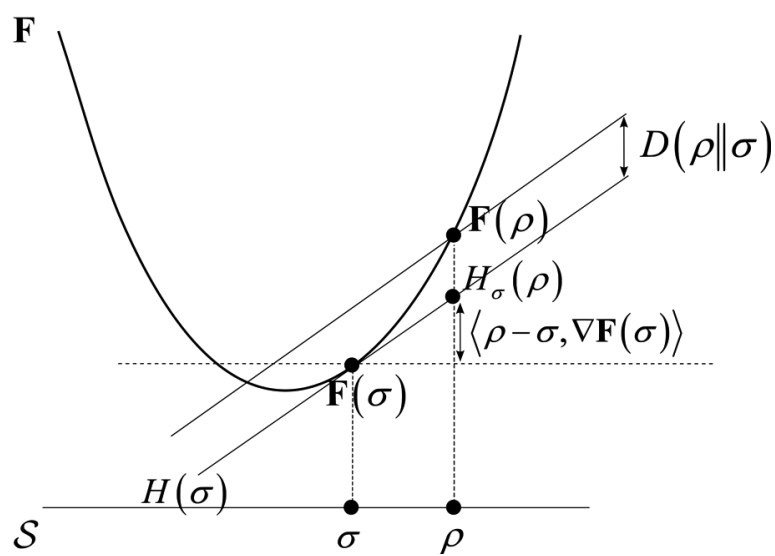

Fig. 10 Depiction of generator function as a negative von Neumann entropy.

relative entropy can be expressed as [10]:

$$
\begin{aligned}
D(\rho \| \sigma)= & \frac{1}{2} \log \frac{1}{4}\left(1-r_{\rho}^{2}\right) \\
& +\frac{1}{2} r_{\rho} \log \frac{\left(1+r_{\rho}\right)}{\left(1-r_{\rho}\right)}-\frac{1}{2} \log \frac{1}{4} .
\end{aligned}
$$

The relative entropy of quantum states can be described by a strictly convex and differentiable generator function $\mathbf{F}$ :

$$
\mathbf{F}(\rho)=-\mathrm{S}(\rho)=\operatorname{Tr}(\rho \log \rho),
$$

where $-\mathrm{S}$ is the negative entropy of quantum states. The quantum relative entropy $D(\rho \| \sigma)$ for density matrices $\rho$ and $\sigma$ is given by generator function $\mathbf{F}$ in the following way [24]:

$$
D(\rho \| \sigma)=\mathbf{F}(\rho)-\mathbf{F}(\sigma)-\langle\rho-\sigma, \nabla \mathbf{F}(\sigma)\rangle,
$$

where $\langle\rho, \sigma\rangle=\operatorname{Tr}\left(\rho \sigma^{*}\right)$ is the inner product of quantum states and $\nabla \mathbf{F}(\cdot)$ is the gradient [24], [29], [30].

In Fig. 10, we have depicted the quantum informational distance, $D(\rho \| \sigma)$, as the vertical distance between the generator function $\mathbf{F}$ and $H(\sigma)$, the hyperplane tangent to $\mathbf{F}$ at $\sigma$. The point of intersection of quantum state $\rho$ on $H(\sigma)$ is denoted by $H_{\sigma}(\rho)$ [24], [28], [29].

As it has been shown by Nielsen et al. [24], [28], for the quantum informational distance function, the generator function is the negative von Neumann entropy function $-\mathrm{S}$,

$$
\mathbf{F}(\rho)=-\mathrm{S}(\rho)=\operatorname{Tr}(\rho \log \rho),
$$

where $F: S\left(\mathbb{C}^{d}\right) \rightarrow \mathbb{R}$. The quantum informational distance function $D_{\mathbf{F}}(\rho \| \sigma)$ with generator function $\mathbf{F}(\rho)=$ $-\mathrm{S}(\rho)$ is illustrated in Fig. 11 [24]. 


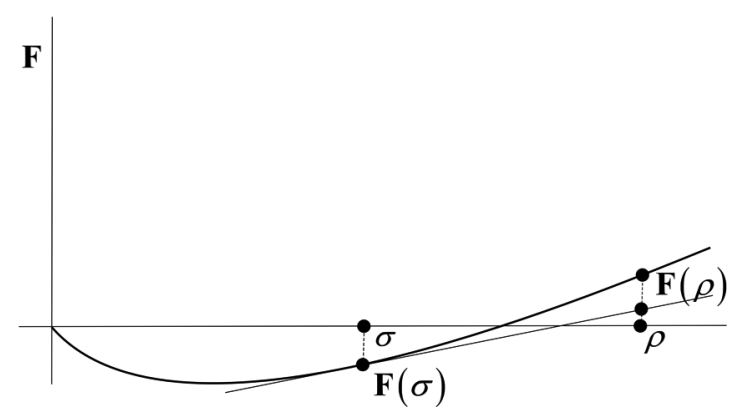

Fig. 11 Negative von Neumann generator function.
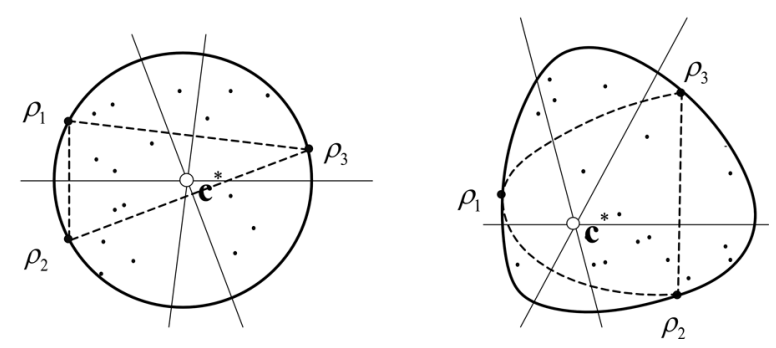

Fig. 12 Circumcenter for Euclidean distance and quantum relative entropy.
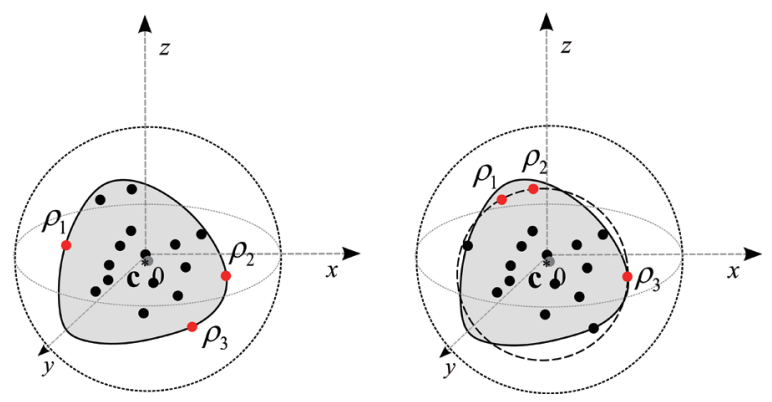

Fig. 13 The maximal distance states of the smallest balls differ for the quantum informational distance and Euclidean distance.

The generator function of the quantum informational distance is the negative von Neumann entropy function. The quantum informational distance function is a linear operator, thus for convex functions $\forall \mathbf{F}_{1} \in C$ and $\forall \mathbf{F}_{2} \in C, D_{\mathbf{F}_{1}+\lambda \mathbf{F}_{2}}(\rho \| \sigma)=D_{\mathbf{F}_{1}}(\rho \| \sigma)+\lambda D_{\mathbf{F}_{2}}(\rho \| \sigma)$, for any $\lambda \geq 0$. The density matrices of quantum states can be represented by $3 \mathrm{D}$ points in the Bloch ball. The geometrical structure of quantum informational balls differs from the geometrical structure of ordinary Euclidean balls.

In Fig. 12, we have illustrated the circumcenter $\mathbf{c}^{*}$ of $\mathcal{S}$ for the Euclidean distance and for quantum relative entropy [24], [29], [30].
In Fig. 13, we compare the smallest quantum informational ball and the ordinary Euclidean ball.

We conclude that the quantum states $\rho_{1}, \rho_{2}$ and $\rho_{3}$ which determine the smallest enclosing ball in a $\mathrm{Eu}-$ clidean geometry differ from the states of the quantum informational ball. This fact also reveals the difference between the mathematical and geometrical background of the two approaches [28].

\subsection{Computation of quantum delaunay triangulation on bloch sphere}

We would like to compute the informationtheoretical radius $r^{*}$ of the smallest enclosing ball of the channel output quantum states which describes the maximal capacity of the channel, thus we must first seek the center $\mathbf{c}^{*}$ of the set of quantum states $\mathcal{S}$. The set $\mathcal{S}$ of quantum states is denoted by $\mathcal{S}=\left\{\rho_{i}\right\}_{i=1}^{n}$. The distance $d(\cdot, \cdot)$ between any two quantum states of $\mathcal{S}$ is measured by the quantum relative entropy, thus a minimax mathematical optimization is applied to the quantum relative entropy-based distances to find the center c of the set $\mathcal{S}$. We denote the quantum relative entropy from $\mathbf{c}$ to the furthest point of $\mathcal{S}$ by $d(\mathbf{c}, \mathcal{S})=$ $\max _{i} d\left(\mathbf{c}, \rho_{i}\right)$. Using a minimax optimization, we can minimize the maximal quantum relative entropy from $\mathbf{c}$ to the furthest point of $\mathcal{S}$ by $\mathbf{c}^{*}=\arg \min _{\mathbf{c}} d(\mathbf{c}, \mathcal{S})$.

In classical computational geometry, Voronoi diagrams and Delaunay triangulations play an important role [13], [16]. A Voronoi diagram is a division of space [28]-[30]. The dual diagram for a Voronoi diagram is called a Delaunay tessellation [13], [14]. In the graph of a Delaunay triangulation, any circle is empty if it contains no vertex of $\mathcal{S}$ in its interior. If two quantum states of set $\mathcal{S}$ are denoted by $\rho$ and $\sigma$, then edge $e$ is in $\operatorname{Del}(\mathcal{S})$ if and only if there exists an empty circle that passes through $\rho$ and $\sigma$. An edge satisfying the empty circle property is said to be Delaunay. The Delaunay triangulation is guaranteed to be a triangulation only if the vertices of $\mathcal{S}$ are in a general position, thus there are no four quantum states of $\mathcal{S}$ lying on the same circle. The circumcircle of a triangle is the unique circle that passes through all three of its vertices, and the triangle is Delaunay if and only if its circumcircle is empty. The quantum Delaunay triangulation of a set of quantum states $\mathcal{S}$, denoted by $\operatorname{Del}(\mathcal{S})$, is the geometric dual of quantum Voronoi diagrams $v o(\mathcal{S})$.

In Fig. 14, we compare a classical Euclidean Delaunay and a quantum informational Delaunay triangulation for a set of quantum states.

The quantum Voronoi diagrams can be first-type or right-sided diagrams. Similarly, we can derive two triangulations from quantum Voronoi diagrams [24], [29], [30]. The first-type quantum informational ball circumscribing any simplex of quantum Delaunay tri- 

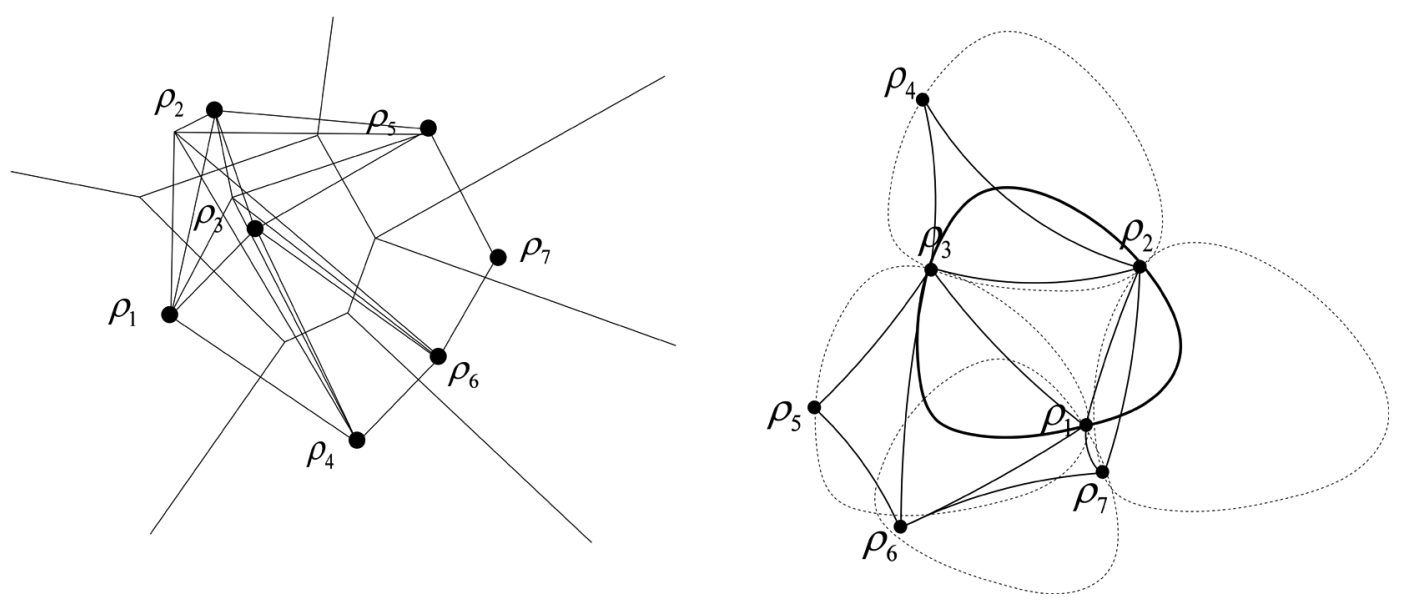

Fig. 14 Comparison of classical Euclidean (a) and quantum Delaunay triangulation (b).
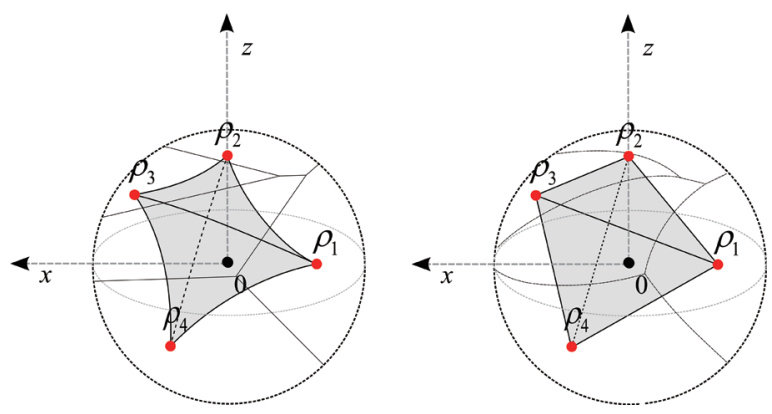

Fig. 15 Comparison of first-type and second-type quantum Delaunay triangulations on the Bloch-ball.

angulation $\operatorname{Del}(\mathcal{S})$ is empty. If we choose a subset $\Upsilon$ of at most $d+1$ states in $\mathcal{S}=\left\{\rho_{1}, \ldots, \rho_{n}\right\}$, then the convex hull of the associated quantum states $\rho_{i}, i \in \Upsilon$, is a simplex of the quantum triangulation of $\mathcal{S}$, iff there exists an empty quantum informational ball $B$ passing through the $\rho_{i}, i \in \Upsilon$. The first-type and second-type quantum diagrams for quantum states which have nonequal radii, are different [24], [29], [30].

In Fig. 15, we compare the first-type and second-type quantum Delaunay diagrams for mixed quantum states on the Bloch sphere.

The approximated value of the radius of the superball is obtained by the radii of the smallest information balls.

\subsection{Laguerre diagram for quantum states}

We use the Laguerre Delaunay diagram [13], [14], [24] to compute the radius of the smallest enclosing ball. In general, the Laguerre distance for generating points $x_{i}$ with weight $r_{i}^{2}$, in a Euclidean space is defined by

$$
d_{L}\left(\rho, x_{i}\right)=\left\|\rho-x_{i}\right\|^{2}-r_{i}^{2} .
$$

The Delaunay diagram for the Laguerre distance is called the Laguerre-Delaunay diagram. As it has been shown by Nielsen et al. [24], for the Laguerre bisector of two three-dimensional Euclidean balls $B\left(\rho, r_{P}\right)$ and $B\left(\sigma, r_{Q}\right)$ centered at quantum states $\rho$ and $\sigma$, we can write the equation

$$
2\langle x, \sigma-\rho\rangle+\langle\rho, \rho\rangle-\langle\sigma, \sigma\rangle+r_{Q}^{2}-r_{P}^{2}=0 .
$$

In a Euclidean space, the Laguerre distance $d_{L}\left(\rho, x_{i}\right)$ with weight $r_{i}^{2}$ can be interpreted as the square of the length of the line segment starting at $\rho$ and tangent to the circle centered at $x_{i}$ with radius $\sqrt{r_{i}^{2}}$. Thus, the circle centered at $x_{i}$ with radius $\sqrt{r_{i}^{2}}$ is the circle associated with $x_{i}$ [13], [14], [24].

We show a new method for deriving the quantum relative entropy-based Delaunay tessellation on the Blochball to analyze the superactivation property of the quantum channel. In our algorithm we present an effective solution to seek the center $\mathbf{c}$ of the set of smallest enclosing quantum information ball, using Laguerre diagrams [13], [14], [24].

Our geometrical analysis has two main steps:

1. We construct Delaunay triangulation from Laguerre diagrams on the Bloch-ball.

2. We seek the center of the smallest enclosing ball.

\subsection{Quantum delaunay triangulation from laguerre di- agrams}

In a Euclidean space, the Laguerre distance of a point $x$ to a Euclidean ball $b=b(\rho, r)$ is defined as $d_{L}(\rho, x)=$ 


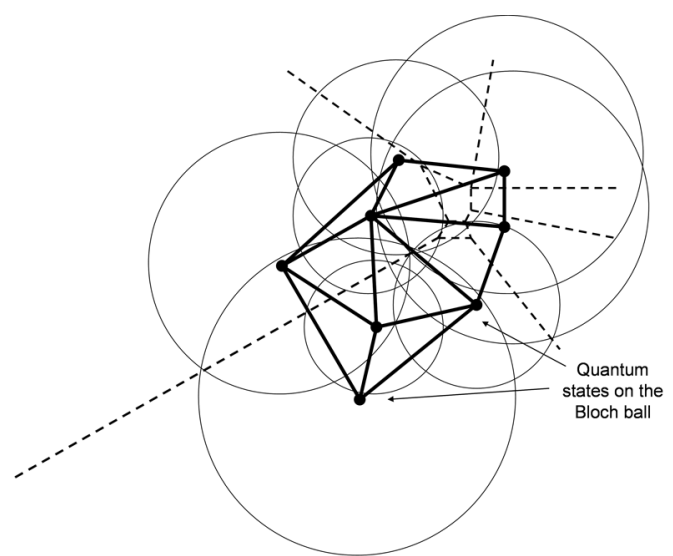

Fig. 16 Regular triangulation on the Bloch-ball.

$\|\rho-x\|^{2}-r^{2}$, and for $n$ balls $b_{i}=b\left(\rho_{i}, r_{i}\right)$, where $i=1, \ldots, n$, the Laguerre diagram [13], [14], [24] of $b_{i}$ is defined as the minimization diagram of the corresponding $n$ distance function

$$
d_{L}^{i}(x)=\|\rho-x\|^{2}-r^{2} .
$$

In Fig. 16, we show the ordinary triangulation of quantum relative entropy-based diagram. As it can be concluded from this result, the computation of quantum Delaunay diagram can be taken back to the classical Euclidean geometry [24], [29], [30].

We use the result of Aurenhammer to construct the quantum relative entropy-based Delaunay tessellation, using the Laguerre diagram of the $n$ Euclidean spheres of equations [14]

$$
\left\langle x-\rho_{i}^{\prime}, x-\rho_{i}^{\prime}\right\rangle=\left\langle\rho_{i}^{\prime}, \rho_{i}^{\prime}\right\rangle+2\left(\mathbf{F}\left(\rho_{i}^{\prime}\right)-\left\langle\rho_{i}, \rho_{i}^{\prime}\right\rangle\right) .
$$

The most important result of this equivalence is that we can efficiently construct a quantum relative entropybased Delaunay triangulation on the Bloch sphere, using fast methods for constructing classical Euclidean Laguerre diagrams [14].

\subsection{Center of the quantum informational ball}

We use an approximation algorithm from classical computational geometry to determine the smallest enclosing ball of balls using core-sets. The core-sets have an important role in our calculation and approximate method. We apply the approximation algorithm presented by Badoui et al. [15], however in our algorithm the distances between quantum states are measured by quantum relative entropy. The $\mathcal{E}$-core set $C$ is a subset of the set $C \subseteq \mathcal{S}$, such that for the circumcenter $\mathbf{c}$ of the minimax ball [15], [22], [23], [25]

$$
d(\mathbf{c}, \mathcal{S}) \leq(1+\mathcal{E}) r
$$

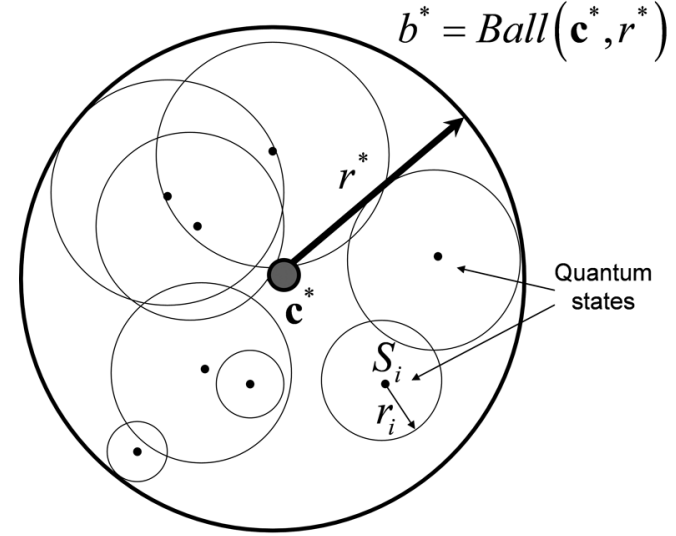

Fig. 17 The smallest enclosing ball of a set of balls in the quantum space.

where $r$ is the radius of the smallest enclosing quantum information ball of the set of quantum states $\mathcal{S}$ [15], [22]. The approximating algorithm, for a set of quantum states $S=\left\{s_{1}, \ldots, s_{n}\right\}$ and circumcenter c, first finds the farthest point $s_{m}$ of ball set $B$, and moves c towards $s_{m}$ in $O(d n)$ time in every iteration step.

The algorithm seeks the farthest point in the ball set $B=\left\{b_{1}=\operatorname{Ball}\left(\mathbf{c}_{1}, r_{1}\right), \ldots, b_{n}=\operatorname{Ball}\left(\mathbf{c}_{n}, r_{n}\right)\right\}$ by maximizing the quantum informational distance for a current circumcenter position $\mathbf{c}$ as $\max _{i \in\{1, \ldots, n\}} D_{F}\left(\mathbf{c}, b_{i}\right)$. Using equation $\max _{x \in b_{i}} D_{F}\left(\mathbf{c}, x_{i}\right)=D_{F}\left(\mathbf{c}, S_{i}\right)+r_{i}$, we get

$$
\left.\max _{i \in\{1, \ldots, n\}} D_{F}\left(\mathbf{c}, b_{i}\right)=\max _{i \in\{1, \ldots, n\}}\right)\left(D_{F}\left(\mathbf{c}, S_{i}\right)+r_{i}\right) .
$$

In Fig. 17, we illustrate the smallest enclosing ball of balls in the quantum space.

We denote the set of $n d$-dimensional balls by $B=$ $\left\{b_{1}, \ldots, b_{n}\right\}$, where $b_{i}=\operatorname{Ball}\left(S_{i}, r_{i}\right), S_{i}$ is the center of ball $b_{i}$ and $r_{i}$ is the radius of the $i$-th ball. The smallest enclosing ball of set $B=\left\{b_{1}, \ldots, b_{n}\right\}$ is the unique ball $b^{*}=\operatorname{Ball}\left(\mathbf{c}^{*}, r^{*}\right)$ with minimum radius $r^{*}$ and center $\mathbf{c}^{*}$ [23], [25]. The algorithm does $\left\lfloor\frac{1}{\mathcal{E}^{2}}\right\rfloor$ iterations to ensure an $(1+\mathcal{E})$ approximation, thus the overall cost of the algorithm is $O\left(\frac{d n}{\mathcal{E}^{2}}\right)$ [15]. The smallest enclosing ball of ball set $B$ can be written as

$$
\min _{\mathbf{c}} \mathbf{F}_{B}(\mathbf{c}),
$$

where $\mathbf{F}_{B}(X)=d(X, B)=\max _{i \in\{1, \ldots, n\}} d\left(X, B_{i}\right)$ and the distance function $d(\cdot, \cdot)$ measures the relative entropy between quantum states [15], [22], [43]. The minimum ball of the set of balls is unique, thus the circumcenter $\mathbf{c}^{*}$ of the set of quantum states is $\mathbf{c}^{*}=\arg \min _{\mathbf{c}} \mathbf{F}_{B}(\mathbf{c})$.

The main steps of our algorithm can be summarized [29], [30]: 
Algorithm 1.

1. Select a random center $\mathbf{c}_{1}$ from the set of quantum states $\mathcal{S}$

$$
\begin{aligned}
& \text { for }\left(i=1,2, \ldots,\left\lceil\frac{1}{\mathcal{E}^{2}}\right\rceil\right) \\
& \text { do }
\end{aligned}
$$

2. Find the farthest point $s$ of $\mathcal{S}$ wrt. quantum relative entropy

$$
S \leftarrow \arg \max _{s^{\prime} \in \mathcal{S}} D_{F}\left(\mathbf{c}_{i}, s^{\prime}\right)
$$

3. Update the circumcircle:

$$
\mathbf{c}_{i+1} \leftarrow \nabla_{F}^{-1}\left(\frac{i}{i+1} \nabla_{F}\left(\mathbf{c}_{i}\right)+\frac{1}{i+1} \nabla_{F}(S)\right) .
$$

4. Return $\mathbf{c}_{i+1}$

At the end of our algorithm, the radius $r^{*}$ of the smallest enclosing ball with respect to the quantum informational distance is equal to the single use classical capacity of the quantum channel.

\section{Fitting the smallest quantum infor- mational ball}

Geometrically, the smallest quantum informational ball can be computed from the intersection of contours of the quantum relative entropy ball with the ellipsoid of the channel, whose ellipsoid is generated by the map of the channel. The maximum length, radius, $\mathbf{r}_{\rho}$, can be determined by an iterative algorithm using the quantum relative entropy as a distance measure. The steps of the approximation of the quantum informational superball are based on the fitting mechanism of a single informational, which will be presented later in this section. In the case of the representation of our superball, the average state and the optimal output state are interpreted from the meaning of joint channel capacity, which states are available after the joint measurement. In the case of the single Bloch ball representation there is average and optimal output state which comes from a single channel measurement [10].

The computation of quantum channel capacity using geometrical approaches also has been presented by Hayashi et al. [41], [42]. Hayashi's work is a very useful and practical tool to approach the capacity of a quantum channel based on the quantum relative entropy function, however in our work we will construct advanced algorithmic approaches to analyze the classical capacity of quantum channels.

In Fig. 18 (a), the smallest quantum informational ball with radius $r^{*}=D_{\max }\left(\mathbf{r}_{\rho} \| \mathbf{r}_{\sigma}\right)$ intersects the channel ellipsoid at magnitude $m_{\rho}$ of the Bloch vector $\mathbf{r}_{\rho}$. The Euclidean distance between the origin and center c $^{*}$ is denoted by $m_{\sigma}$. Similarly, the Euclidean distance between the origin and state $\rho$ is denoted by $m_{\rho}$. In our geometrical iteration algorithm, we would like to

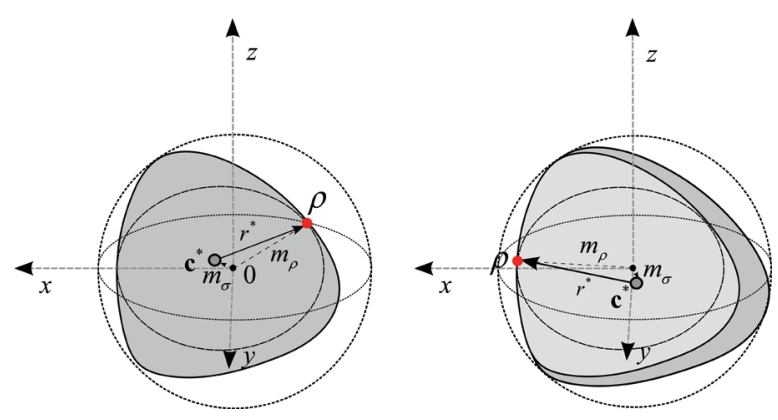

Fig. 18 Intersection of radius of smallest enclosing quantum informational ball and channel ellipsoid (a). The optimal ball is shown in light-grey (b).

determine the location of vector $\mathbf{r}_{\sigma}$ inside the channel ellipsoid such that, the largest possible contour value $D_{\max }\left(\mathbf{r}_{\rho} \| \mathbf{r}_{\sigma}\right)$ touches the channel ellipsoid surface and the remainder of the $D_{\max }$ contour surface lies entirely outside the channel ellipsoid. The point on the channel ellipsoid surface is defined as the set of channel output $\rho$. The vector $\mathbf{r}_{\sigma}$ is defined in the interior of the ellipsoid, as the convex hull of the channel ellipsoid. To determine the optimal length of the radius, the algorithm moves point $\sigma$.

As we move vector $\mathbf{r}_{\sigma}$ from the optimum position, a larger contour corresponding to the larger value of the quantum relative entropy $D$ will intersect the channel ellipsoid surface, thereby $\max _{\mathbf{r}_{\rho}} D\left(\mathbf{r}_{\rho} \| \mathbf{r}_{\sigma}\right)$ will increase. We can conclude that vector $\mathbf{r}_{\sigma}$ should be adjusted to minimize $\max _{\mathbf{r}_{\rho}} D\left(\mathbf{r}_{\rho} \| \mathbf{r}_{\sigma}\right)$, as illustrated in Fig. 18 (b).

The vector $\mathbf{r}_{\sigma}$ should be adjusted to minimize the value of $\max _{\mathbf{r}_{\rho}} D\left(\mathbf{r}_{\rho} \| \mathbf{r}_{\sigma}\right)$. To find the optimal value of vector $\mathbf{r}_{\sigma}$ in our geometrical analysis, we choose a start point for vector $\mathbf{r}_{\sigma}$ in the interior of the ellipsoid.

In Fig. 19 (a), we show the initial start point inside the channel ellipsoid chosen by the algorithm. The vector of state $\sigma$ is denoted by $\mathbf{r}_{\sigma}$. In the next step, the algorithm determines the set of points to the vector $\mathbf{r}_{\rho}^{\prime}$ on the ellipsoid surface most distant from $\mathbf{r}_{\sigma}$, using the quantum relative entropy as distance measure. In Fig. 19 (b), the new state is notated by $\rho^{\prime}$.

The maximum distance between the states can be expressed as $\max _{\mathbf{r}_{\rho}} D\left(\mathbf{r}_{\rho}^{\prime} \| \mathbf{r}_{\sigma}\right)$. We choose a random Bloch sphere vector from the maximal set of points according to vector $\mathbf{r}_{\rho}^{\prime}$. The selected point is denoted by $\mathbf{r}_{\rho}^{\prime \prime}$. The algorithm makes a step from $\mathbf{r}_{\sigma}$ towards the surface point vector $\mathbf{r}_{\rho}^{\prime \prime}$ in the Bloch sphere space. In this step, the algorithm updates vector $\mathbf{r}_{\sigma}$ to

$$
\mathbf{r}_{\sigma}^{*}=(1-\gamma) \mathbf{r}_{\sigma}+\gamma \mathbf{r}_{\rho}^{\prime \prime},
$$

where $\gamma$ denotes the size of the step. In Fig. 20 (a), the updated state and the vector of the state are denoted by 

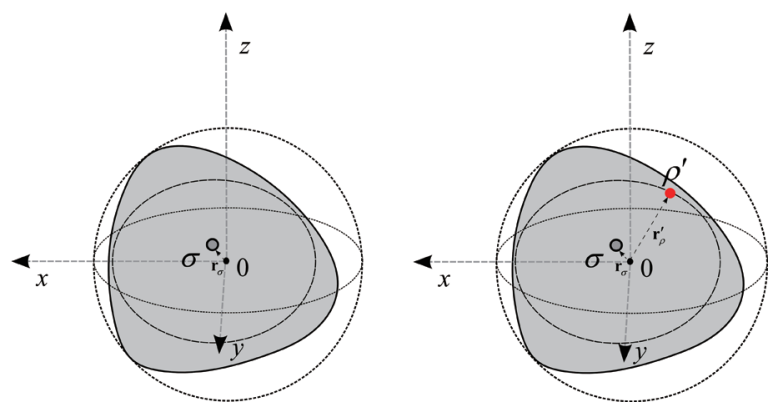

Fig. 19 The algorithm determines the points on the ellipsoid surface most distant from the point, using the quantum relative entropy as distance measure.
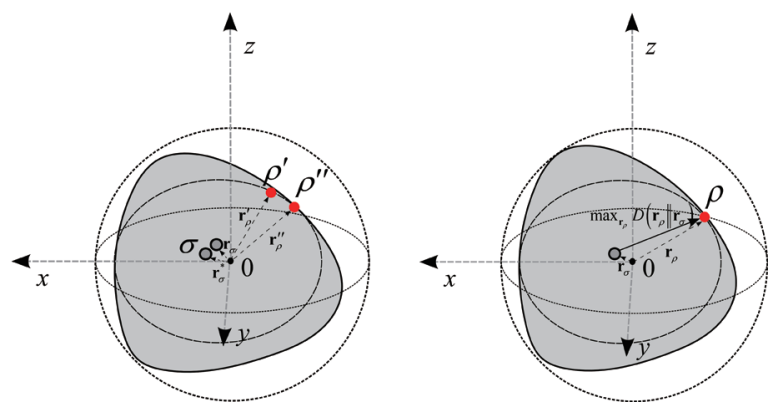

Fig. 20 The algorithm makes a step towards the found surface point vector and updates the vector.

$\rho^{\prime \prime}$ and $\mathbf{r}_{\rho}^{\prime \prime}$. The center of the quantum informational ball is denoted by $\mathbf{r}_{\sigma}^{*}$.

In Fig. 20 (b), we illustrate the quantum informational distance between the final center point and the maximal distance state $\rho$, using the notation $\max _{\mathbf{r}_{\rho}} D\left(\mathbf{r}_{\rho} \| \mathbf{r}_{\sigma}\right)$. Using the updated vector $\mathbf{r}_{\sigma}^{*}$, the algorithm continues to loop until $\max _{\mathbf{r}_{\rho}^{*}} D\left(\mathbf{r}_{\rho}^{\prime} \| \mathbf{r}_{\sigma}^{*}\right)$ no longer changes. We conclude that the iteration converges to the optimal $\mathbf{r}_{\sigma}$, because the algorithm minimizes $\max _{\mathbf{r}_{\rho}} D\left(\mathbf{r}_{\rho} \| \mathbf{r}_{\sigma}\right)$.

At the end of the iteration process, the radius of the smallest quantum informational ball can be expressed as

$$
\min \max _{\mathbf{r}_{\rho}} D\left(\mathbf{r}_{\rho} \| \mathbf{r}_{\sigma}\right) .
$$

In our geometrical method, we compute $r^{*}$, the radius of the smallest enclosing quantum informational superball, to determine the information-theoretic superactivation of the quantum channel capacities.

\section{Efficient coreset construction of channel output states}

The coreset technique has deep relevance to classical computational geometry. A coreset of a set of output quantum states has the same behavior as the larger input set, so clustering and other approximations can be made with smaller coresets. The coreset can be viewed as a smaller input set of channel output states, hence it can be used as the input to an approximation algorithm. The weighted sum of errors of the smaller coreset is a $(1 \pm \varepsilon)$ approximation of the larger input set. The bound on this error can be decreased only if the center points that form a finite set are used in the approximation. These coresets are called weak coresets [22], [25] and this method can be applied in quantum space between quantum states.

Using weak coresets, the run time of $(1+\varepsilon)$ coreset algorithms [25] with respect to quantum informational distance can be improved. To construct the coreset method analyzing the superactivation of zero-error capacity quantum channels, we have to introduce the definition of similar quantum informational distances and weak coresets of quantum states.

\subsection{Similar quantum informational distance}

The quantum informational distance is asymmetric and contains singularities, since there exist density matrices $\rho$ and $\sigma$ for which $D(\rho, \sigma)=\infty$. The similar quantum informational divergence function does not contain these singularities and these distances are approximately symmetric. To use the similar quantum informational divergence function, first we define it as follows. The quantum informational distance function $D(\rho \| \sigma)$ between density matrices $\rho$ and $\sigma$ is $\mu$-similar for a positive real constant $\mu$, if there exists a positive definite matrix $A$ such that

$$
\mu D_{A}(\rho \| \sigma) \leq D(\rho \| \sigma) \leq D_{A}(\rho \| \sigma) .
$$

For quantum informational distances, if the domain is given as $\chi=[\lambda, \gamma] \subseteq R_{+}^{d}$, then $\mu=\frac{\lambda}{\gamma}$ and $\frac{1}{2 \lambda} I$. If we have $0<\lambda<\gamma$, then the quantum informational distance function can be calculated by the $D(\rho \| \sigma)$ quantum relative entropy function on the domain $\chi=[\lambda, \gamma] \subseteq R^{d}[25]$. As can be proven, the quantum informational distance is $\mu$-similar if $\mu=\frac{\lambda}{\gamma}$ and $A=\frac{1}{2 \lambda} I$. In these cases, the quantum informational distance function is $\mu$-similar, because it is restricted to a sub-domain, which avoids the singularities [19]. It can be easily proven that the quantum informational distance function is strictly convex and all second-order partial derivates exist and are continuous on the domain $\chi=[\lambda, \gamma] \subseteq R^{d}$ with parameters $\mu=\frac{\lambda}{\gamma}$ and $A=\frac{1}{2 \lambda} I[19]$. The applied coreset algorithm was 
originally presented by Chen et al. [20], [21]. We show that this method can be used to generate a coreset based on a similar quantum informational distance function. To obtain an enhanced version of previously known coreset approximation algorithms, we must define weak coresets.

\subsection{Weak coreset of quantum states}

The weak coresets include all the relevant information required to analyze the original extremely large input set. The coreset approach has significantly lower computational complexity, hence it can be applied very efficiently [19], [20]. In our method, weak coresets are applied to $\mu$-similar quantum informational distances since, in these subsets, the distances between quantum states are symmetric, hence singularities can be avoided and fast Euclidean methods can be applied. To analyze the properties of quantum channels, we use the same method as presented in [19]. We use this subset to approximate the original input set, with approximately $(1 \pm \varepsilon)$ error, hence we can put forward the following statement.

The superactive quantum channels can be discovered with approximation error $(1 \pm \varepsilon)$, by using the smaller $\mu$-similar subset of input quantum states.

Using the results of Chen's work [20], we show that by using this algorithm, the superactivation capability of quantum channels can be approximated with error $(1 \pm \varepsilon)$, using the smaller $\mu$-similar subset of input quantum states. Applying Chen's modified algorithm, the $(1 \pm \varepsilon)$-approximation can be obtained in run time $O(d k n)$, where $k$ is the number of medians of channel output quantum states. The goal of the algorithm is to find a set of size $k$ such that the sum of errors of quantum informational distances is minimized, hence $\operatorname{error}\left(\rho_{i}, \sigma\right)=\sum_{i=1}^{n} \min D_{\sigma}\left(\rho_{i} \| \sigma\right)$. The algorithm solves the $k$-median problem with respect to the quantum informational distance $D_{\sigma}$ in quantum space. The output of the algorithm is a set of $k$ quantum states, for which the function $\operatorname{error}\left(\rho_{i}, \sigma\right)$ is minimized. We generalize the $k$-median problem for quantum informational distances. Let us assume that we have two quantum states $\rho$ and $\sigma$ in domain $\mathcal{S}$. We would like to construct a subset of $\mathcal{S}_{\text {OUT }}$ of $k$ quantum states, for which

$$
D\left(\rho, \mathcal{S}_{\text {OUT }}\right)=\min _{\sigma \in \mathcal{S}^{*}} D(\rho \| \sigma) .
$$

The $k$-median problem for quantum states can be stated as follows. We would like to use only a finite set $\mathcal{S}_{I N}$ of quantum states from the original larger space. For a set $\mathcal{S}_{I N}$, we would like to construct a set $\mathcal{S}_{\text {OUT }}$ of $k$-quantum states, for which $\operatorname{error}\left(\mathcal{S}_{I N}, \mathcal{S}_{\text {OUT }}\right)=$

$$
\begin{aligned}
& \sum_{\rho \in \mathcal{S}_{I N}} D_{\sigma}\left(\rho \| \mathcal{S}_{\text {OUT }}\right) \text { is minimized, hence } \\
& \operatorname{error}\left(\mathcal{S}_{I N}, \mathcal{S}^{*}\right)=\sum_{\rho \in \mathcal{S}_{I N}} \min D_{\sigma}\left(\rho \| \mathcal{S}^{*}\right) .
\end{aligned}
$$

The error of the optimal solution for input states $\mathcal{S}_{I N}$ is denoted by $\operatorname{opt}_{k}\left(\mathcal{S}_{I N}\right)$, and the elements of the output set $\mathcal{S}_{\text {OUT }}$ are the $k$ median-quantum states of set $\mathcal{S}_{I N}$. To construct a more efficient algorithm, we use only the $\mu$-similar quantum informational distances, hence the set of input quantum states $\mathcal{S}_{I N}$ is restricted to quantum states for which the singularities can be avoided [19].

The superactivation properties of quantum channels can be discovered by using $\mu$-similar quantum informational distances and the coreset construction method.

For any set $\mathcal{S}_{I N}$ of size $n$ quantum states and for any finite $\mathcal{W} \subseteq \mathcal{S}$, there exists a weak coreset of size $O\left(\frac{1}{\varepsilon^{2}} k \log (n) \log \left(k|\mathcal{W}|^{k} \log n\right)\right)$. This $\mathcal{W}$-weak coreset of quantum states can be constructed in time $O\left(\frac{1}{\varepsilon^{2}} k \log (n) \log \left(k|\mathcal{W}|^{k} \log n\right)+d k n\right)$, where $k$ is the number of quantum states in set $\mathcal{S}_{\text {OUT }}, n$ is the number of input states and $d$ is the dimension of the points. The previous result can be integrated into our analysis as follows.

Using $\mu$-similar quantum informational distances and the $\mathcal{W}$-weak coreset of quantum states, the superactivation of quantum channels can be analyzed by an $(1+\varepsilon)$-approximation algorithm in a run time $O\left(d^{2} 2^{k / \varepsilon} \log ^{k+2} n+d k n\right)$. With the result of Banerjee et al. [27], the optimal 1-median of any given input set $\mathcal{S}$ in quantum space can be uniquely defined by the centroid $c=\frac{1}{|\mathcal{S}|} \sum_{\rho \in \mathcal{S}} \rho$

Using the fact that an optimal solution of the $k$ median clustering problem can be approached by $(k-1)$ linearly separable subsets, it can be shown that for any set $\mathcal{S}_{I N}$, at most $n^{d k}$ states have to considered as one of the optimal $k$-median quantum states of $\mathcal{S}_{I N}$ [20], [21]. We use a smaller set $\mathcal{S}$ from $\mathcal{S}_{I N}$, which is a small weighted set that has the same clustering behavior as the larger input set $\mathcal{S}_{I N}$. The coreset method used in our approach can be defined by the error of the approximation in terms of the quantum informational distance between quantum states as follows:

$$
\operatorname{error}_{w}\left(\mathcal{S}, \mathcal{S}_{\text {OUT }}\right)=\sum_{\rho \in \mathcal{S}} w(\rho) D\left(\rho \| \mathcal{S}_{\text {OUT }}\right),
$$

and this error is a $(1 \pm \varepsilon)$-approximation of $\operatorname{error}\left(\mathcal{S}_{I N}, \mathcal{S}_{\text {OUT }}\right)$ for any set of quantum states $\mathcal{S}_{\text {OUT }}$ of size $\left|\mathcal{S}_{\text {OUT }}\right|=k$. For the weak coreset construction, 
let us assume that we have a set of quantum states $\mathcal{S}_{I N}$ and a set $\mathcal{W}$. If the weight function is defined by

$$
\sum_{S} w(\rho)=\left|\mathcal{S}_{I N}\right|
$$

then the weighted set $\mathcal{S}$ is a $\mathcal{W}$-weak coreset of $\mathcal{S}_{I N}$, iff for all $\mathcal{S}_{\text {OUT }} \in \mathcal{W}$ of size $\left|\mathcal{S}_{\text {OUT }}\right|=k$, we have

$$
\begin{aligned}
& \left|\operatorname{error}\left(\mathcal{S}_{I N}, \mathcal{S}_{O U T}\right)-\operatorname{error}_{w}\left(\mathcal{S}, \mathcal{S}_{O U T}\right)\right| \\
& \quad \leq(\varepsilon) \operatorname{error}\left(\mathcal{S}_{I N}, \mathcal{S}_{O U T}\right) .
\end{aligned}
$$

This $\mathcal{W}$-weak coreset is called the $(k, \varepsilon)$ weak-coreset of $\mathcal{S}_{I N}$. To get this construction with this error bound, we use the results of Chen [20].

\subsubsection{Coreset method for quantum informational dis- tances}

To apply the modified coreset method, we have to construct a $[\alpha, \beta]$ bicriteria approximation algorithm to get the set of median quantum states $M=$ $\left\{\sigma_{1}, \sigma_{2}, \ldots \sigma_{k}\right\}$ of a $k$-median clustering of $\mathcal{S}_{I N}$, for which $\operatorname{error}\left(\mathcal{S}_{I N}, M\right) \leq \operatorname{\alpha opt}_{k}\left(\mathcal{S}_{I N}\right)$ and $|M|=k \leq \beta k$. Using the results of [19], [25] the bicriteria algorithm can be summarized as follows:

\section{Bicriteria algorithm to channel analysis}

1. Choose an initial quantum state $\sigma_{1}$ uniformly at random from $\mathcal{S}_{I N}$

2. Let $M$ be the set of chosen quantum states from $\mathcal{S}_{I N}$. State $\rho \in \mathcal{S}_{I N}$ is chosen with probability $\frac{D(\rho \| M)}{\operatorname{error}\left(\mathcal{S}_{I N}, M\right)}$ as next state of $M$.

3. Repeat step 2 until $M$ contains $k$ quantum states.

At the end of the bicriteria algorithm, we have a set of median quantum states $M=\left\{\sigma_{1}, \sigma_{2}, \ldots \sigma_{k}\right\}$, for which $\operatorname{error}\left(\mathcal{S}_{I N}, M\right) \leq \operatorname{\alpha opt}_{k}\left(\mathcal{S}_{I N}\right)$ and $|M|=k \leq \beta k$. After application of the bicriteria algorithm, we use the modified coreset construction method presented in [20] to the quantum states as follows:

\section{Coreset algorithm to quantum channel analysis}

1. Partition $\mathcal{S}_{I N}$ into $\mathcal{S}_{1}, \mathcal{S}_{2} \ldots, \mathcal{S}_{k}$ by assuming each quantum state $\rho \in \mathcal{S}_{I N}$ to their closest $\sigma_{i} \in M$

2. Let $\rho \in \mathcal{S}_{I N}$ iff $\sigma_{i}=\arg \min _{\sigma \in M} D(\rho \| \sigma)$.

3. Let $R=\frac{1}{\alpha n} \operatorname{error}\left(\mathcal{S}_{I N}, M\right)$.

4. Define quantum informational ball $\mathcal{B}\left(\sigma_{i}\right)$ with radius $r^{*}$ and center $\sigma_{i}$ as follows:

$\mathcal{B}\left(\sigma_{i}\right)=D\left(x \| \sigma_{i}\right) \leq r$

5. Define partition of $\left\{\mathcal{S}_{i j}\right\}_{i, j}$ of $\mathcal{S}_{I N}$ by $\mathcal{S}_{i j}=\mathcal{S}_{i} \cap \mathcal{B}\left(\sigma_{i}\right)$ for $i=1,2, \ldots, k$

6. Let $\mathcal{S}_{i j}=\mathcal{S}_{i} \cap\left(\mathcal{B}_{2^{j}}\left(\sigma_{i}\right) \backslash \mathcal{B}_{2^{j-1}}\left(\sigma_{i}\right)\right)$ for $i=1,2, \ldots, k$ and $j=i=1,2, \ldots \gamma$, where $\gamma=\lceil\log (\alpha n)\rceil$.
7. For $i, j$ let $\mathcal{S}_{i j}$ be a uniform set from $\mathcal{S}_{I N}$ of size $\left|\mathcal{S}_{i j}\right|=m$. 8. Let $w(\rho)=\frac{1}{m}\left|\mathcal{S}_{i j}\right|$ be the weight associated with $\rho \in \mathcal{S}_{i j}$.

9. Define weak coreset $\mathcal{S}$ of input quantum states $\mathcal{S}_{I N}$ as follows:

$$
\mathcal{S}=\bigcup_{i, j} \mathcal{S}_{i j} \text { of size }|\mathcal{S}|=m k \gamma=m \beta k\lceil\log (\alpha n)\rceil .
$$

As shown by Ackermann et al. [19], if $m=$ $\Omega\left(\frac{\alpha^{2}}{\varepsilon^{2}} \log \left(\frac{\beta}{\delta} k|\mathcal{W}|^{k} \log (\alpha n)\right)\right)$, then the output set $\mathcal{S}=$ $\cup \mathcal{S}_{i j}$ of Chen's algorithm [20] is a $\mathcal{W}$-weak coreset of $\mathcal{S}_{I N}$ with probability $1-\delta$. As also shown in [19], if we have found the parameters $[\alpha, \beta]$ with the bicriteria algorithm, then an $[\alpha, \beta]$-approximate $k$ median clustering of $\mathcal{S}_{I N}$ in a $\mathcal{W}$-weak coreset of size $O\left(\frac{1}{\varepsilon^{2}} k \log n \log \left(k|\mathcal{W}|^{k} \log n\right)\right)$ can be constructed in time $O\left(d k n+\frac{1}{\varepsilon^{2}} k \log n \log \left(k|\mathcal{W}|^{k} \log n\right)\right)$.

Using Chen's coreset construction [20], we design an algorithm for clustering quantum states, using the previously generated $\mathcal{W}$-weak coreset of set $\mathcal{S}_{I N}$. The algorithm has approximation error $(1+\varepsilon)$, however the run time of the proposed method is more efficient since it uses the $\mathcal{W}$-weak coreset of set $\mathcal{S}_{I N}$ generated by Chen's algorithm, instead of the original input set $\mathcal{S}_{I N}$. For clustering of quantum states, we use the modified version of Ackermann's method from [19]. As can be concluded using the modified coreset and clustering algorithm and the $\mathcal{W}$-weak coreset of the original input set $\mathcal{S}_{I N}$, the superactivation properties of quantum channels can be analyzed very efficiently. The $(1+\varepsilon)$-approximation can be obtained in a run time $O\left(d^{2} 2^{k / \varepsilon} \log ^{k+2} n+d k n\right)$

\subsection{Determination of median-quantum states}

The clustering method of [19] for a weak set of quantum states and $\mu$-similar quantum informational distances can be summarized as follows:

\section{CLUSTER : Clustering of channel output states}

1. Let $\mathcal{S}_{I N}$ the set of remaining input states, with $w\left(\mathcal{S}_{I N}\right)=n$

2. Let the weight function on input quantum states $\mathcal{S}_{I N}$

3. Let $m$ the number of median-quantum states yet to be found

4. Let $C$ the set of medians already found

5. if $m=0$ then return $C$

6. else

7. $\quad$ if $m \geq\left|\mathcal{S}_{I N}\right|$ then return $C \cup \mathcal{S}_{I N}$

8. else

9. Sample a multiset of quantum states $\mathcal{M}$

$$
\text { of size } \frac{96 k^{2}}{\varepsilon^{2} \mu \delta} \text { from } \mathcal{S}_{I N}
$$



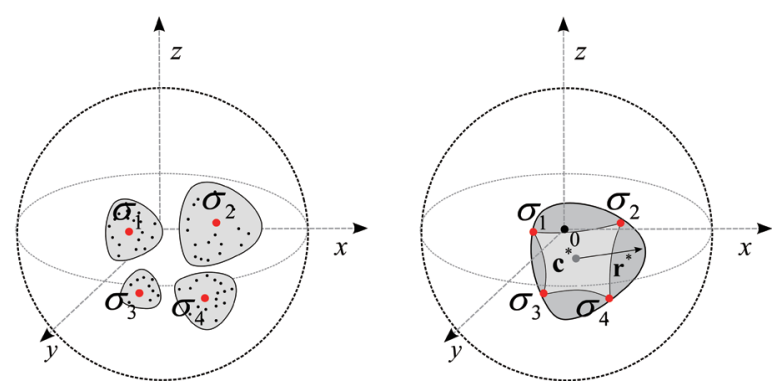

Fig. 21 Clustering of quantum states. The smallest quantum informational ball contains the computed medians.

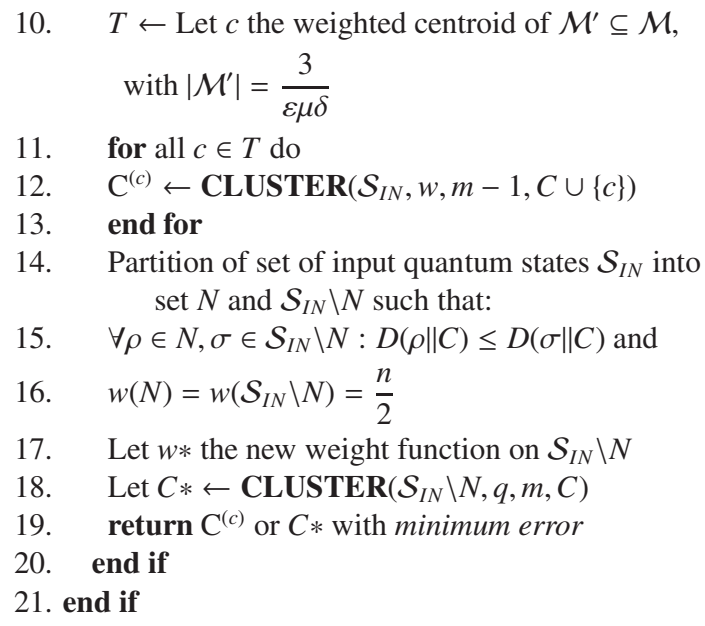

In Fig. 21, we illustrate the clustering of channel output states. In the clustering process, our algorithm computes the median-quantum states denoted by $\sigma_{i}$, using a fast weak coreset and clustering algorithm. In the next step, we compute the convex hull of the median quantum states and, from the convex hull, the radius of the smallest quantum informational ball can be obtained. The smallest superball measures the channel capacity, hence the radius of the superball is equal to the sum of radii of quantum balls of independent channel outputs. The output states are measured by a joint measurement setting.

As shown in Fig. 22 the proposed analysis combines the weak coreset method of Chen and the clustering algorithm presented by Ackermann. To use Chen's method [20], [21] to construct the weak coreset, we apply a bicreteria algorithm [19], [20] to find the required parameters, then we apply the result of Ackermann et al. [19] in quantum space. In both methods, we use quantum informational distance functions as distance measures. With the help of the coreset method, we can construct a more efficient $(1+\varepsilon)$-approximation algorithm in quantum space, using only a small subset of

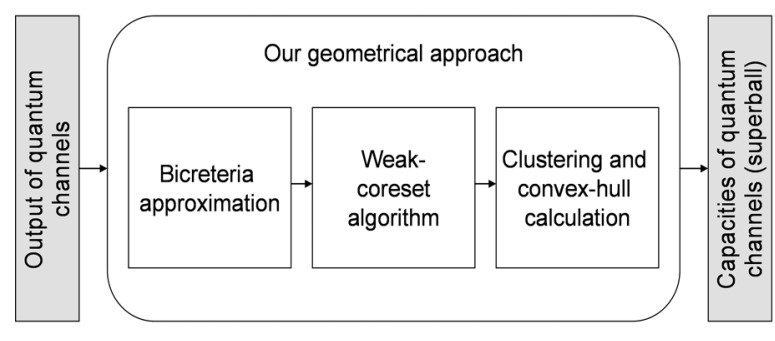

Fig. 22 Decomposition of our geometrical approach. The output of the algorithm is the radius of the superball.

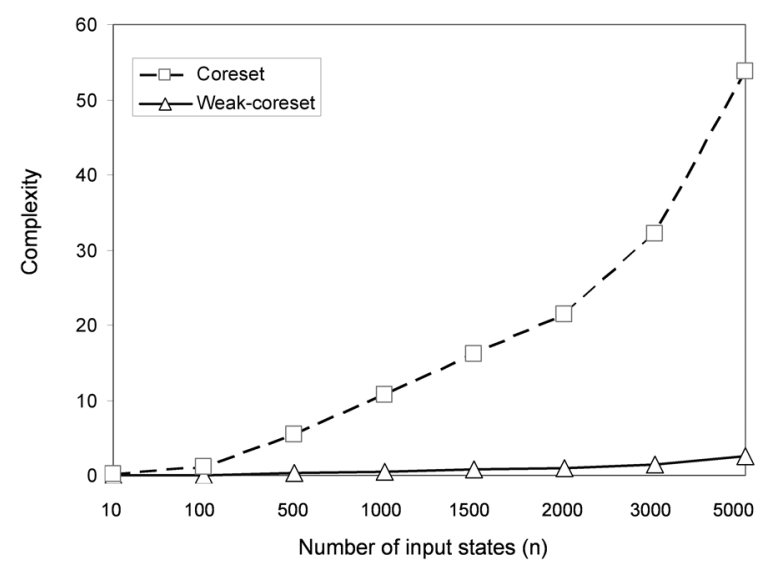

Fig. 23 Complexity of coreset and weak coreset algorithms for clustering channel output quantum states.

the original larger input set.

Using the modified weak coreset algorithm and the $(1+\varepsilon)$-approximation algorithm, the superactivation of quantum channels can be analyzed relative to $\mu$ similar quantum informational distances and $k$ medianquantum states with error $\operatorname{error}\left(\mathcal{S}_{I N}, \mathcal{S}_{O U T}\right) \leq(1+$ $7 \varepsilon) \operatorname{opt}_{k}\left(\mathcal{S}_{I N}\right)$.

The overall run time of the algorithm used above has been proven to be $O\left(d^{2} 2^{k / \varepsilon} \log ^{k+2} n+d k n\right)$, with constant probability, since the working mechanism of the proposed method is based on probabilistic events.

The bicreteria algorithm can be computed in time $O(d k n)$, hence the coreset method can be constructed in time $O(|\mathcal{W}|+d k n)$.

In Fig. 23, we have compared the complexity of the coreset algorithm presented in [25] with our advanced weak coreset approach, as a function of input size. Both algorithms result in $(1+\varepsilon)$-approximations, however the complexities of the proposed methods differ significantly.

As our results confirm, the complexity of the weak coreset method is significantly lower than for the coreset method, especially for a large number of input quan- 
tum states $n$. The precision of the approximation is the same as the standard coreset approach, however the complexity of our weak coreset method is significantly lower, hence the quantum channels can be analyzed more efficiently.

\section{Conclusions and future work}

This paper shows a fundamentally new algorithmic solution for superactivation of the asymptotic zeroerror capacity of quantum channels. With the help of the proposed informational geometric approach, the complexity of the computation of the zero-error capacity of the quantum channels can be dramatically decreased. Using our method, a larger set of superactive zero-capacity channels can be discovered very efficiently and our method can bridge the gap between theoretical and experimental results. The proposed algorithmic solution can be the key to finding other possible channel models and channel parameter domains, with possible combinations being proved by theory. If there exist other combinations of channel models that realize superactivation of the classical zero-error capacity of the quantum channels, our method can find them. We have constructed an extremely fast recursive geometric algorithm to find the conditions for the computation and for the superactivation of the asymptotic classical zero-error capacity of the quantum channels.

This paper is intended as an introduction to our algorithmical framework to study the superactivation of the classical zero-error capacity of quantum channels. Our method is the first to solve the problem of the computation of classical zero-error capacity of quantum channels.

In future work, we would like to show some explicit results of the combinations of channels used to realize the superactivation of the classical zero-error capacity of quantum channels. We would like to extend our method to analyze the superactivation of the asymptotic quantum capacity of zero-capacity quantum channels, and we would like to implement and verify our results in practice, using zero-capacity optical quantum channels.

\section{Acknowledgement}

The authors would like to thank the financial support from "Sandor Csibi" Ph.D. Researcher Scholarship at the Budapest University of Technology, Faculty of Electrical Engineering and Informatics, Hungary.

\section{References}

[1] G. Smith and J. Yard, "Quantum Communication with Zero-capacity Channels," Science vol.321, pp.18121815, 2008.

[2] R. Duan, "Superactivation of zero-error capacity of noisy quantum channels," arXiv:0906.2527 [quant-ph], 2009

[3] T. Cubitt, J. X. Chen, and A. Harrow, arXiv: 0906.2547.

[4] R. Duan, Y. Feng, Z. F. Ji, and M. S. Ying, Phys. Rev. Lett., vol.98, p.230502, 2007.

[5] T. Cubitt, A. Montanaro, and A. Winter, arXiv: 0706.0705 [quant-ph], 2007.

[6] T. Cubitt, A. Harrow, D. Leung, A. Montanaro, and A. Winter, Comm. Math. Phys., vol.284, no.1, p.281, 2008.

[7] R. C. Medeiros and F. M. de Assis, Int. J. Quant. Inf., vol.3, p.135, 2005

[8] R. C. Medeiros, R. Alleaume, G. Cohen, and F. M. de Assis, quant-ph/0611042.

[9] S. Beigi and P. W. Shor, arXiv:0709.2090 [quant-ph], 2007.

[10] J. A. Cortese, "The Holevo-Schumacher-Westmoreland Channel Capacity for a Class of Qudit Unital Channels," 2002, LANL ArXiV e-print quant-ph/0211093.

[11] S. Imre and F. Balázs, Quantum Computing and Communications - An Engineering Approach, John Wiley and Sons Ltd, 2005.

[12] M. B. Ruskai, S. Szarek, and E. Werner, "An Analysis of Completely-Positive Trace-Preserving Maps on 2 by 2 Matrices," 2001, LANL ArXiV e-print quant$\mathrm{ph} / 0101003$.

[13] J.-D. Boissonnat, C. Wormser, and M. Yvinec, Curved Voronoi diagrams, In J.-D. Boissonnat and M. Teillaud Eds, Effective Computational Geometry for Curves and Surfaces, pp.67-116, Springer-Verlag, Mathematics and Visualization, 2007.

[14] F. Aurenhammer and R. Klein, Voronoi Diagrams, In J. Sack and G. Urrutia Eds, Handbook of Computational Geometry, Chapter V, pp.201-290, Elsevier Science Publishing, 2000.

[15] M. Badoiu, S. Har-Peled, and P. Indyk, "Approximate clustering via core-sets," In Proceedings 34th ACM Symposium on Theory of Computing, pp.250-257, 2002.

[16] B. W. Schumacher and M. Westmoreland, "Relative Entropy in Quantum Information Theory" 2000, LANL ArXiV e-print quant-ph/0004045.

[17] B. W. Schumacher and M. Westmoreland, "Optimal Signal Ensembles," 1999, LANL ArXiV e-print quant$\mathrm{ph} / 9912122$.

[18] L. Gyongyosi and S. Imre, "Novel Geometrical Solution to Additivity Problem of Classical Quantum Channel Capacity," The 33rd IEEE Sarnoff Symposium - 2010, Princeton University, Apr. 2010, Princeton, New Jersey, USA.

[19] M. R. Ackermann, J. Blömer, and C. Sohler, "Clustering for metric and non-metric distance measures," In Proceedings of the 19th Annual ACM-SIAM Symposium on Discrete Algorithms (SODA '08), pp.799-808. Society for Industrial and Applied Mathematics, 2008. 
[20] K. Chen, "On k-median clustering in high dimensions," In Proceedings of the 17th Annual ACM-SIAM Symposium on Discrete Algorithms (SODA '06), pp.11771185, 2006.

[21] K. Chen, "On k-median and k-means clustering in metric and Euclidean spaces and their applications," Manuscript, URL: http://ews.uiuc.edu/'kechen/, July 2007.

[22] G. Frahling and C. Sohler, "Coresets in dynamic geometric data streams," In Proceedings of the 27th Annual ACM Symposium on Theory of Computing (STOC '05), pp.209-217, New York, NY, USA, 2005. ACM.

[23] S. Har-Peled and A. Kushal, "Smaller coresets for kmedian and k-means clustering," In Proceedings of the 21st Annual Symposium on Computational Geometry (SCG '05), pp.126-134, New York, NY, USA, 2005. ACM.

[24] F. Nielsen, J.-D. Boissonnat, and R. Nock, "On Bregman Voronoi diagrams," In Proceedings of the 18th Annual ACM-SIAM Symposium on Discrete Algorithms (SODA '07), pp.746-755, Philadelphia, PA, USA, 2007. Society for Industrial and Applied Mathematics.

[25] D. Feldman, M. Monemizadeh, and C. Sohler, "A PTAS for k-means clustering based on weak coresets," In Proceedings of the 23rd ACM Symposium on Computational Geometry (SCG '07), pp.11-18, 2007.

[26] K. Kato, M. Oto, H. Imai, and K. Imai, "Voronoi diagrams for pure 1-qubit quantum states," quantph/0604101, 2006.

[27] A. Banerjee, S. Merugu, I. S. Dhillon, and J. Ghosh, "Clustering with Bregman divergences," Journal of Machine Learning Research, vol.6, pp.1705-1749, 2005.

[28] R. Nock and F. Nielsen, "Fitting the Smallest Enclosing Bregman Ball,” ECML 2005, pp.649-656, ECML'05.

[29] F. Nielsen and R. Nock, "On the smallest enclosing information disk. Inf. Process," Lett. IPL '08, vol.105, no.3, pp.93-97, 2008.

[30] F. Nielsen and R. Nock, "Quantum Voronoi Diagrams and Holevo Channel Capacity for 1-Qubit Quantum States," ISIT 2008.

[31] F. Nielsen and R. Nock, "Bregman Sided and Symmetrized Centroids," ICPR 2008, ICPR'08, arXiv:0711.3242.

[32] F. Nielsen and R. Nock, "Approximating Smallest Enclosing Balls with Application to Machine Learning," International Journal on Computational Geometry and Applications (IJCGA '09), 2009.

[33] M. B. Hastings, "A Counterexample to Additivity of Minimum Output Entropy" Nature Physics, vol.5, p.255, 2009. arXiv:0809.3972.

[34] F. Brandao and M. Horodecki, "On Hastings' counterexamples to the minimum output entropy additivity conjecture," arXiv:0907.3210.
[35] C. King, "Additivity for unital qubit channels," J. Math. Phys., vol.43, pp.4641-4653, 2002.

[36] M. Fukuda, C. King, and D. K. Moser, "Comments on Hastings' Additivity Counterexamples," Communications in Mathematical Physics, 2010, DOI 10.1007/s00220-010-0996-9.

[37] N. Datta, A. S. Holevo, and Y. Suhov, "A quantum channel with additive minimum output entropy," 2004, URL: http://arxiv.org/abs/quant-ph/0403072.

[38] K. Matsumoto and F. Yura, "Entanglement cost of antisymmetric states and additivity of capacity of some quantum channels," Journal of Phys. A, vol.37, pp.L167-L171, 2004.

[39] P. Shor, "Additivity of the classical capacity of entanglementbreaking quantum channels," J. Math. Phys., vol.246, no.3, pp.453-472, 2004, URL: http://arxiv.org/abs/quant-ph/0305035.

[40] M. M. Wolf and J. Eisert, "Classical information capacity of a class of quantum channels," New Journal of Physics, vol.7, no.93, 2005.

[41] M. Hayashi and H. Nagaoka, "General formulas for capacity of classical-quantum channels," IEEE Transactions on Information Theory, vol.49, no.7, pp.17531768, 2003.

[42] M. Hayashi, H. Imai, K. Matsumoto, M. B. Ruskai and T. Shimono, "Qubit channels which require four inputs to achieve capacity: Implications for additivity conjectures," Quantum Information and Computation, vol.5, pp.032-040, 2005.

[43] L. Gyongyosi and S. Imre, "Computational Geometric Analysis of Physically Allowed Quantum Cloning Transformations for Quantum Cryptography," In Proceedings of the 4th WSEAS International Conference on Computer Engineering and Applications (CEA '10), pp.121-126, Harvard University, Cambridge, USA, 2010.

[44] L. Gyongyosi and S. Imre, "Information Geometrical Solution to Additivity of Non-Unital Quantum Channels," QCMC 2010, 10th Quantum Communication, Measurement \& Computing Conference, July 2010, University of Queensland, Brisbane, Queensland, Australia.

[45] L. Gyongyosi and S. Imre, "Computational Information Geometric Analysis of Quantum Channel Additivity," Photon10 Conference, Quantum Electronics Group (QEP-19), 2010, Southampton, UK.

[46] L. Gyongyosi and S. Imre, "Information Geometrical Analysis of Additivity of Optical Quantum Channels," IEEE/OSA Journal of Optical Communications and Networking (JOCN), IEEE Photonics Society \& Optical Society of America, ISSN: 1943-0620, 2010.

[47] C. Shannon, "The zero-error capacity of a noisy channel," IEEE Trans. Information Theory, pp.8-19, 1956.

[48] B. Bollobas, Modern graph theory, Springer-Verlag New York, Inc., New York, 1998. 
[49] J. Koner and A. Orlitsky, "Zero-error information theory," IEEE Trans. Info. Theory, vol.44, no.6, pp.22072229, 1998.

[50] L. Gyongyosi and S. Imre, "Capacity Recovery of Useless Photonic Quantum Communication Channels," ALS Conference, Lawrence Berkeley National Laboratory (Berkeley Lab), University of California, Berkeley (California), USA, 2010.

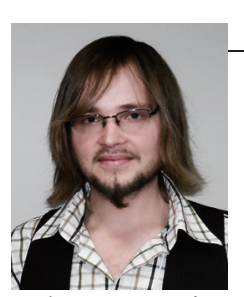

\section{Laszlo GYONGYOSI}

Laszlo GYONGYOSI received the M.Sc. degree in Computer Science with Honors from the Budapest University of Technology and Economics (BUTE) in 2008. He is a final year Ph.D. Candidate at the Department of Telecommunications, BUTE. His research interests are in Quantum Computation and Communication, Quantum Channel Capacity, Quantum Cryptography and Quantum Information Theory. He is currently completing a book on advanced quantum communications, and he teaches courses in Quantum Computation. In 2009, he received Future Computing Best Paper Award on quantum information, in 2010, he was awarded the Best Paper Prize of University of Harvard, USA. In 2010, he obtained a Ph.D. Researcher Grant from University of Arizona, USA.

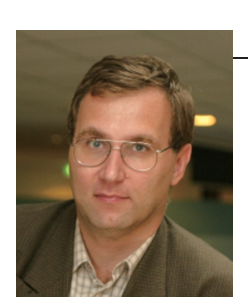

Sandor IMRE

Sandor IMRE was born in Budapest in 1969. He received the M.Sc. degree in Electronic Engineering from the Budapest University of Technology (BUTE) in 1993. Next he started his Ph.D. studies at BUTE and obtained dr. univ. degree in 1996, Ph.D. degree in 1999 and DSc degree in 2007. Currently he is carrying his teaching activities as Head of the Dept. of Telecommunications of BUTE. He was invited to join the Mobile Innovation Centre of BUTE as R\&D director in 2005. His research interest includes mobile and wireless systems, quantum computing and communications. Especially he has contributions on different wireless access technologies, mobility protocols and reconfigurable systems. 